\title{
A Comprehensive Study of the Discovery Potential of NOvA, T2K, and T2HK Experiments
}

\author{
C. Soumya, K. N. Deepthi, and R. Mohanta \\ School of Physics, University of Hyderabad, Hyderabad 500 046, India \\ Correspondence should be addressed to R. Mohanta; rukmani98@gmail.com
}

Received 9 November 2015; Revised 22 January 2016; Accepted 28 January 2016

Academic Editor: Filipe R. Joaquim

Copyright (C) 2016 C. Soumya et al. This is an open access article distributed under the Creative Commons Attribution License, which permits unrestricted use, distribution, and reproduction in any medium, provided the original work is properly cited. The publication of this article was funded by $\mathrm{SCOAP}^{3}$.

\begin{abstract}
With the recent measurement of reactor mixing angle $\theta_{13}$ the knowledge of neutrino oscillation parameters has improved significantly except the CP violating phase $\delta_{\mathrm{CP}}$, mass hierarchy, and the octant of the atmospheric mixing angle $\theta_{23}$. Many dedicated experiments are proposed to determine these parameters which may take at least 10 years from now to become operational. It is therefore very crucial to use the results from the existing experiments to see whether we can get even partial answers to these questions. In this paper we study the discovery potential of the ongoing NO $v \mathrm{~A}$ and T2K experiments as well as the forthcoming T2HK experiment in addressing these questions. In particular, we evaluate the sensitivity of $\mathrm{NO} v \mathrm{~A}$ to determine neutrino mass hierarchy, octant degeneracy, and $\delta_{\mathrm{CP}}$ after running for its scheduled period of 3 years in neutrino mode and 3 years in antineutrino mode. We then extend the analysis to understand the discovery potential if the experiments will run for $(5 v+5 \bar{v})$ years and $(7 v+3 \bar{v})$ years. We also show how the sensitivity improves when we combine the data from $\mathrm{NO} v \mathrm{~A}, \mathrm{~T} 2 \mathrm{~K}$, and T2HK experiments with different combinations of run period. The CP violation sensitivity is marginal for $\mathrm{T} 2 \mathrm{~K}$ and $\mathrm{NO} \nu \mathrm{A}$ experiments even for ten-year data taking of NO $\nu \mathrm{A}$. T2HK has a significance above $5 \sigma$ for a fraction of two-fifths values of the $\delta_{\mathrm{CP}}$ space. We also find that $\delta_{\mathrm{CP}}$ can be determined to be better than $35^{\circ}, 21^{\circ}$, and $9^{\circ}$ for all values of $\delta_{\mathrm{CP}}$ for $\mathrm{T} 2 \mathrm{~K}, \mathrm{NO} v \mathrm{~A}$, and $\mathrm{T} 2 \mathrm{HK}$ respectively.
\end{abstract}

\section{Introduction}

The discovery of neutrino oscillations has firmly established that neutrinos are massive. It has marked the beginning of many neutrino oscillation experiments. The mixing of three neutrino flavors can be described by Pontecorvo-MakiNakagawa-Sakata matrix $U_{\text {PMNS }}[1,2]$, which is parameterized in terms of three rotation angles $\theta_{12}, \theta_{23}$, and $\theta_{13}$ and three $\mathrm{CP}$ violating phases, one Dirac type $\left(\delta_{\mathrm{CP}}\right)$ and two Majorana types $(\rho$ and $\sigma$ ). The neutrino oscillation data accumulated over many years allows us to determine the solar and atmospheric neutrino oscillation parameters with very high precision. Recently, the reactor mixing angle $\theta_{13}$ has been measured precisely [3-7] with a moderately large value, quite close to its previous upper bound. The observation of the largish value of $\theta_{13}$ has attracted a lot of attention in recent times to understand the mixing pattern in the lepton sector. Furthermore, it also opens up promising prospects for the observation of leptonic CP violation $[8,9]$. As $\theta_{13}$ is nonzero, there could be $\mathrm{CP}$ violation in the lepton sector, analogous to the quark sector, provided the $\mathrm{CP}$ violating phase $\delta_{\mathrm{CP}}$ is not vanishingly small.

The current results from recent neutrino oscillation experiments [10-13] and their global analysis [14-17] performed by several groups have implied that the minimal three-neutrino framework is adequate to describe the observed oscillation phenomenology. The best-fit values and the $3 \sigma$ ranges of the oscillation parameters from [17] are presented in Table 1 .

Another important discovery in recent times is the precision measurement of $\sin ^{2} \theta_{23}$ by MINOS experiment [18], which is found to be nonmaximal. Using the complete set of accelerator and atmospheric data they disfavored the maximal mixing by $-2 \Delta \log (\mathscr{L})=1.54$. They obtained the best-fit value for the mixing angle $\theta_{23}$ as $\sin ^{2} \theta_{23}=0.41$, known as lower octant (LO) and $\sin ^{2} \theta_{23}=0.61$, the so-called higher octant $(\mathrm{HO})$ values. 
TABLE 1: The best-fit values and the $3 \sigma$ ranges of the neutrino oscillation parameters from [17].

\begin{tabular}{lcc}
\hline Mixing parameters & Best-fit value & $3 \sigma$ range \\
\hline $\sin ^{2} \theta_{12}$ & 0.323 & $0.278 \rightarrow 0.375$ \\
\hline $\sin ^{2} \theta_{23}(\mathrm{NH})$ & 0.567 & $0.392 \rightarrow 0.643$ \\
$\sin ^{2} \theta_{23}(\mathrm{IH})$ & 0.573 & $0.403 \rightarrow 0.640$ \\
\hline $\sin ^{2} \theta_{13}(\mathrm{NH})$ & 0.0234 & $0.0177 \rightarrow 0.0294$ \\
$\sin ^{2} \theta_{13}(\mathrm{IH})$ & 0.0240 & $0.0183 \rightarrow 0.0297$ \\
\hline$\Delta m_{21}^{2} / 10^{-5} \mathrm{eV}^{2}$ & 7.6 & $7.11 \rightarrow 8.18$ \\
\hline$\left|\Delta m_{31}\right|^{2} / 10^{-3} \mathrm{eV}^{2}(\mathrm{NH})$ & 2.48 & $2.30 \rightarrow 2.65$ \\
$\left|\Delta m_{31}\right|^{2} / 10^{-3} \mathrm{eV}^{2}(\mathrm{IH})$ & 2.38 & $2.20 \rightarrow 2.54$ \\
\hline
\end{tabular}

With these exciting discoveries of nonzero $\theta_{13}$ and nonmaximal $\theta_{23}$, the focus of neutrino oscillation studies has now been shifted towards the determination of other unknown parameters. These include the determination of mass hierarchy, octant of the atmospheric mixing angle $\theta_{23}$, discovery of $\mathrm{CP}$ violation, and the magnitude of the $\mathrm{CP}$ violating phase $\delta_{\mathrm{CP}}$. In this paper we would like to investigate the prospects of addressing these issues with the off-axis longbaseline experiments $\mathrm{T} 2 \mathrm{~K}, \mathrm{NO} v \mathrm{~A}$, and $\mathrm{T} 2 \mathrm{HK}$ with updated experimental specifications. The experimental specifications of these experiments are briefly described below.

(i) $\mathrm{NO} v \mathrm{~A}[19,20]$ is an off-axis long-baseline neutrino oscillation experiment designed to study $\nu_{\mu} \rightarrow$ $v_{e}$ appearance measurements using Fermilab NuMI muon neutrino beam $\left(v_{\mu}\right)$. Its secondary aim is to precisely measure $\nu_{\mu}$ disappearance parameters. It uses a high intensity proton beam with a beam power of $0.7 \mathrm{MW}$ with $6 \times 10^{20} \mathrm{POT} /$ year. Its detector is a 14 kt totally active liquid scintillator detector (TASD) located at Ash River, $810 \mathrm{~km}$ from Fermilab. The detector is located slightly off the centerline (14 mrad) to the neutrino beam where one can find a large flux of neutrinos of $2 \mathrm{GeV}$ energy. The oscillation from $v_{\mu} \rightarrow v_{e}$ is expected to be maximum at this energy. It is scheduled to run 3 years in $v$ mode followed by 3 years in $\bar{v}$ mode. The detector properties of $\mathrm{NO} v \mathrm{~A}$ considered in our simulations are taken from [21] with the following characteristics as given in Table 2.

(ii) T2K (Tokai-to-Kamiokande) is a currently running long-baseline experiment designed to study neutrino oscillations. An intense $v_{\mu}$ beam of $0.77 \mathrm{MW}$ power is directed from J-PARC to Super-Kamiokande detector, $295 \mathrm{~km}$ away. It has a $22.5 \mathrm{kt}$ water Cherenkov detector. The details of T2K experiment can be found in [22]. We have considered input files for T2K from the General Long-Baseline Experiment Simulator (GLoBES) package [22-24] and the updated experimental description of T2K is taken from [25]. We have matched our results with the ones presented in [25]. In this analysis, we have used $(3 \nu+2 \bar{v})$ running modes for $\mathrm{T} 2 \mathrm{~K}$.

(iii) T2HK (Tokai-to-Hyper-Kamiokande) is a future long-baseline experiment which is expected to be operational around 2023. It can be considered a natural advancement to the ongoing $\mathrm{T} 2 \mathrm{~K}$ experiment. It has the same baseline and off-axis angle as T2K experiment. It uses J-PARC's neutrino experimental facilities with an improved beam power (7.5 MW) and $1 \mathrm{Mt}$ volume water Cherenkov detector, HyperKamiokande (Hyper-K). We have considered a fiducial volume of $0.56 \mathrm{Mt}$ and beam power of $7.5 \mathrm{MW}$ and other specifications are taken from [26]. Hence, T2HK will have high statistics of neutrino events compared to T2K. These features of T2HK make it one of the most sensitive experiment to probe neutrino $\mathrm{CP}$ violation. The input files for T2HK are obtained from GLoBES package [22-24]. The primary objective of this experiment is the discovery of $\mathrm{CP}$ asymmetry.

Since these experiments use $v_{\mu}$ beam and also will run in antineutrino mode their main focus is to study the appearance $\left(v_{\mu} \rightarrow v_{e}\right)$ and the disappearance channels $\left(\nu_{\mu} \rightarrow\right.$ $v_{\mu}$ ) along with their antineutrino counterparts. Since the leading term in the appearance channels $\nu_{\mu} \rightarrow \nu_{e}\left(P_{\mu e}\right)$ and the corresponding antineutrino mode $\bar{\nu}_{\mu} \rightarrow \bar{\nu}_{e}\left(P_{\bar{\mu} \bar{e}}\right)$ is proportional to $\sin ^{2} 2 \theta_{13} \sin ^{2} \theta_{23}$ and with the observation of moderately large value of $\theta_{13}$, these experiments are wellsuited for the determination of mass hierarchy and the octant of $\theta_{23}$. Although the ongoing $\mathrm{NO} v \mathrm{~A}$ and T2K experiments are not planned to measure $\delta_{\mathrm{CP}}$ or to explore $\mathrm{CP}$ violation in the neutrino sector, we would like to investigate whether it is possible to constrain the $\delta_{\mathrm{CP}}$ phase using the data from these two experiments. In other words, how much of the $\delta_{\mathrm{CP}}$ space can be ruled out by these experiments within the next 10 years? In particular, we would like to investigate

(i) whether the combination of $\mathrm{T} 2 \mathrm{~K}(3+2)$ and $\mathrm{NO} v \mathrm{~A}$ $(3+3)$ provide more quantitative answer on the above posed questions than each one of these experiments,

(ii) how the sensitivity on $\delta_{\mathrm{CP}}$, mass hierarchy, and $\theta_{23}$ octant will improve if $\mathrm{NO} v \mathrm{~A}$ runs for 10 years in the $(5+5)$ and $(7+3)$ combination of modes,

(iii) the sensitivities of T2HK experiment for its scheduled run of 3 years in neutrino and 7 years in antineutrino mode.

The paper is organized as follows. In Section 2 we briefly describe the physics reach of these experiments. The prospect of octant resolution and mass hierarchy determination are discussed in Sections 3 and 4. Section 5 contains the CP violation discovery potential and the correlations between the $\mathrm{CP}$ violating phase $\delta_{\mathrm{CP}}$ and the mixing angles $\theta_{13}$ and $\theta_{23}$. We summarize our results in Section 6.

\section{Physics Reach}

As discussed before, the determination of the mass hierarchy, octant of the atmospheric mixing angle $\theta_{23}$ and the search for $\mathrm{CP}$ violation in the neutrino sector are the important physics goals of the current and future oscillation experiments. A simple way to achieve the above three goals is to measure the oscillation probabilities $P\left(v_{\mu} \rightarrow v_{e}\right)$ and $P\left(\bar{\nu}_{\mu} \rightarrow \bar{v}_{e}\right)$. This can 
TABLE 2: Details of NO $v$ A detector characteristics considered in this analysis.

\begin{tabular}{lc}
\hline Signal efficiency & $45 \%$ for $\nu_{e}$ and $\bar{\nu}_{e}$ signal \\
& $100 \% \nu_{\mu}$ CC and $\bar{\nu}_{\mu}$ CC \\
\hline \multirow{2}{*}{ Background efficiency } & $0.83 \% \nu_{\mu}$ CC, $0.22 \% \bar{\nu}_{\mu}$ CC \\
& $2 \% \nu_{\mu}$ NC, $3 \% \bar{\nu}_{\mu}$ NC \\
& $26 \%(18 \%) \nu_{e}\left(\bar{\nu}_{e}\right)$ beam contamination \\
\hline NC background smearing & Migration matrices \\
\hline Systematics & $5 \%$ signal normalization error \\
& $10 \%$ background normalization error \\
\hline
\end{tabular}

be seen from the expression for probability of oscillation from $\nu_{\mu}\left(\bar{\nu}_{\mu}\right) \rightarrow \nu_{e}\left(\bar{\nu}_{e}\right)$ [27-29], where we have kept terms only first order in $\sin \theta_{13}$ and $\alpha=\Delta m_{21}^{2} / \Delta m_{31}^{2}$ :

$$
\begin{aligned}
P\left(\nu_{\mu} \longrightarrow v_{e}\right) \approx \sin ^{2} 2 \theta_{13} \sin ^{2} \theta_{23} \frac{\sin ^{2}(\widehat{A}-1) \Delta}{(\widehat{A}-1)^{2}}+\alpha \\
\cdot \cos \theta_{13} \sin 2 \theta_{12} \sin 2 \theta_{13} \sin 2 \theta_{23} \\
\\
\cdot \frac{\sin \widehat{A} \Delta}{\widehat{A}} \frac{\sin (\widehat{A}-1) \Delta}{(\widehat{A}-1)} \\
\cdot \cos \left(\Delta+\delta_{\mathrm{CP}}\right),
\end{aligned}
$$

where $\Delta m_{i j}^{2}=m_{i}^{2}-m_{j}^{2}, \Delta=\Delta m_{31}^{2} L / 4 E$, and $\widehat{A}=2 \sqrt{2} G_{F} n_{e} E /$ $\Delta m_{31}^{2} . G_{F}$ is the Fermi coupling constant and $n_{e}$ is the electron number density. The transition probability can be enhanced or suppressed depending on the oscillation parameters $\theta_{13}$, $\theta_{23}$, mass hierarchy, that is, the sign of $\Delta m_{31}^{2}$ and CP violation phase $\delta_{\mathrm{CP}}$. Parameters $\alpha, \Delta$, and $\widehat{A}$ are sensitive to neutrino mass ordering. For neutrinos, $\widehat{A}$ is positive for normal hierarchy $(\mathrm{NH})$ and negative for inverted hierarchy (IH), while its sign changes when we go from neutrino to antineutrino mode. Moreover, sign of $\delta_{\mathrm{CP}}$ is reversed for antineutrinos.

It should be noted from (1) that the leading term in the transition probability $P\left(\nu_{\mu} \rightarrow v_{e}\right)$ is proportional to $\sin ^{2} 2 \theta_{13} \sin ^{2} \theta_{23}$. Therefore, the observed moderately large value of $\theta_{13}$ makes it possible for the current generation longbaseline experiments to address the problems of hierarchy and the octant of $\theta_{23}$ determination. The second term in (1) shows the prominence of matter effect on the oscillation probability. The dependency of all the terms on a moderately large reactor neutrino mixing angle $\theta_{13}$ suggests that $\mathrm{NO} \nu \mathrm{A}$ detector will be able to collect a good number of $\nu_{\mu}\left(\bar{\nu}_{\mu}\right) \rightarrow$ $\nu_{e}\left(\bar{v}_{e}\right)$ events.

First, we will try to see whether the energy spectrum information will help us in resolving the octant degeneracy and mass hierarchy. We use GLoBES package $[30,31]$ for the simulation to obtain the energy spectra. In our analysis, we consider the true values of oscillation parameters as provided in Table 3, unless mentioned otherwise.

Figure 1 shows the energy spectrum of the appearance probabilities $P\left(\nu_{\mu} \rightarrow v_{e}\right)$ for neutrino (left panel) and $P\left(\bar{\nu}_{\mu} \rightarrow\right.$
TABLE 3: The true values of oscillation parameters considered in the simulations.

\begin{tabular}{lc}
\hline $\sin ^{2} \theta_{12}$ & 0.32 \\
\hline $\sin ^{2} 2 \theta_{13}$ & 0.1 \\
\hline $\sin ^{2} \theta_{23}$ & $0.41(\mathrm{LO}), 0.59(\mathrm{HO})$ \\
\hline$\Delta m_{\mathrm{atm}}^{2}$ & $2.4 \times 10^{-3} \mathrm{eV}^{2}$ for NH \\
& $-2.4 \times 10^{-3} \mathrm{eV}^{2}$ for IH \\
\hline$\Delta m_{21}^{2}$ & $7.6 \times 10^{-5} \mathrm{eV}^{2}$ \\
\hline$\delta_{\mathrm{CP}}$ & $0^{\circ}$ \\
\hline
\end{tabular}

$\bar{\nu}_{e}$ ) for antineutrino (right panel) for $\mathrm{NO} v \mathrm{~A}$ experiment, where we have varied $\delta_{\mathrm{CP}}$ within the range $-\pi$ to $\pi$. In the upper panel the red (blue) band is for $\mathrm{NH}$ (IH). Furthermore, in each band the probability for $\delta_{\mathrm{CP}}=90^{\circ}$ and $\delta_{\mathrm{CP}}=-90^{\circ}$ cases are shown explicitly by the magenta and green lines. Due to matter effect the probability $P_{\mu e}$ increases for $\mathrm{NH}$ and decreases for IH and vice versa for $P_{\bar{\mu} \bar{e}}$. Thus, for $\delta_{\mathrm{CP}}$ lying in the lower half plane (LHP), that is, $-180^{\circ} \leq \delta_{\mathrm{CP}} \leq 0$, $P_{\mu e}$ is larger and, for $\delta_{\mathrm{CP}}$ in the upper half plane (UHP) $(0 \leq$ $\left.\delta_{\mathrm{CP}} \leq 180^{\circ}\right), P_{\mu e}$ is much lower. The situations reverse for the antineutrino probability $P_{\bar{\mu} \bar{e}}$. Thus, LHP is the favorable half plane for NH and UHP is for IH for neutrino mode. However, the most unfavorable condition is $\left(\mathrm{NH}, \delta_{\mathrm{CP}}=90^{\circ}\right)$ and $(\mathrm{IH}$, $\delta_{\mathrm{CP}}=-90^{\circ}$ ) as the bands almost overlap with each other for the entire energy range. In the lower panels of Figure 1, we show the energy spectrum of $P_{\mu e}$ and $P_{\bar{\mu} \bar{e}}$ for two different values of $\theta_{23}$ assuming $\mathrm{NH}$ to be true hierarchy. The blue band in both of the panels is for $\theta_{23}$ in the LO and red band is for $\theta_{23}$ in the HO. As can be seen from the figures the two bands overlap with each other for some values of $\delta_{\mathrm{CP}}$ and are distinct for others. The overlap regions are the unfavorable ones for the determination of the $\theta_{23}$ octant.

\section{Octant Resolution as a Function of $\theta_{23}$}

In this section we present the results of our analysis on octant sensitivity of $\theta_{23}$ for $\mathrm{T} 2 \mathrm{~K}, \mathrm{NO} \nu \mathrm{A}$, and $\mathrm{T} 2 \mathrm{HK}$ experiments. We also show the results when the data from all the experiments are combined. Although the octant sensitivity of various long-baseline experiments has been discussed extensively by many authors [32-34], here we would like to revisit the octant resolution potential of these experiments with the updated specification details. For $\mathrm{NO} v \mathrm{~A}$, we consider its scheduled $(3 v+3 \bar{v})$ years of run, for T2K $(3 v+2 \bar{v})$ years of run, and for T2HK $(3 v+7 \bar{v})$ years of run. Furthermore, we also investigate the following situation: if $\mathrm{NO} \nu \mathrm{A}$ continues to run for the next 10 years what would be the potential for resolving octant degeneracy for $(5 v+5 \bar{v})$ as well as $(7 v+3 \bar{v})$ years of running? We also see the synergy between $\mathrm{T} 2 \mathrm{~K}, \mathrm{NO} \nu \mathrm{A}$, and $\mathrm{T} 2 \mathrm{HK}$ experiments for their scheduled runs.

The indistinguishability of $\theta_{23}$ and $\left(\pi / 2-\theta_{23}\right)$ is known as octant degeneracy. The relevant oscillation probability 


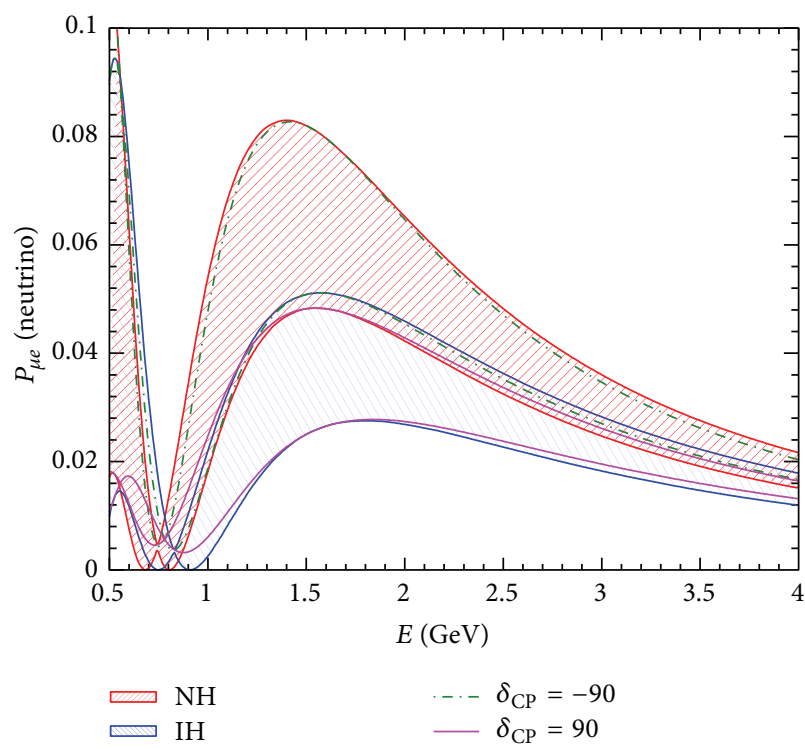

(a)

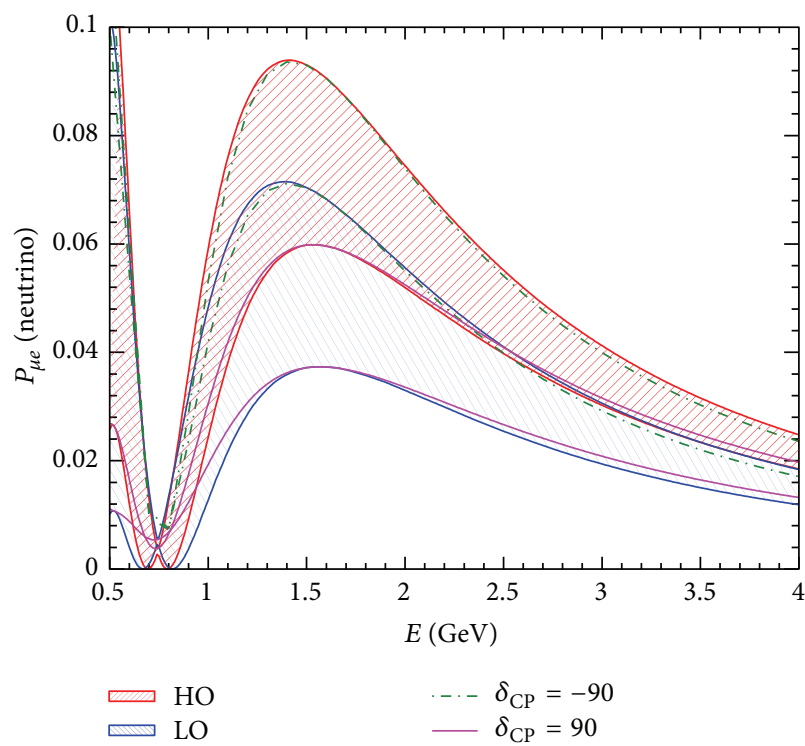

(c)

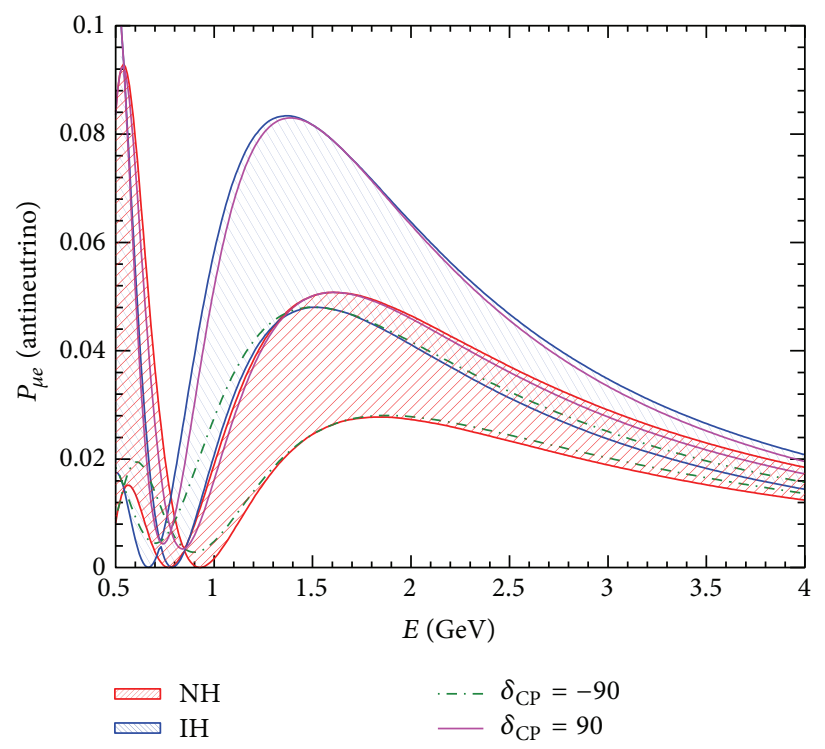

(b)

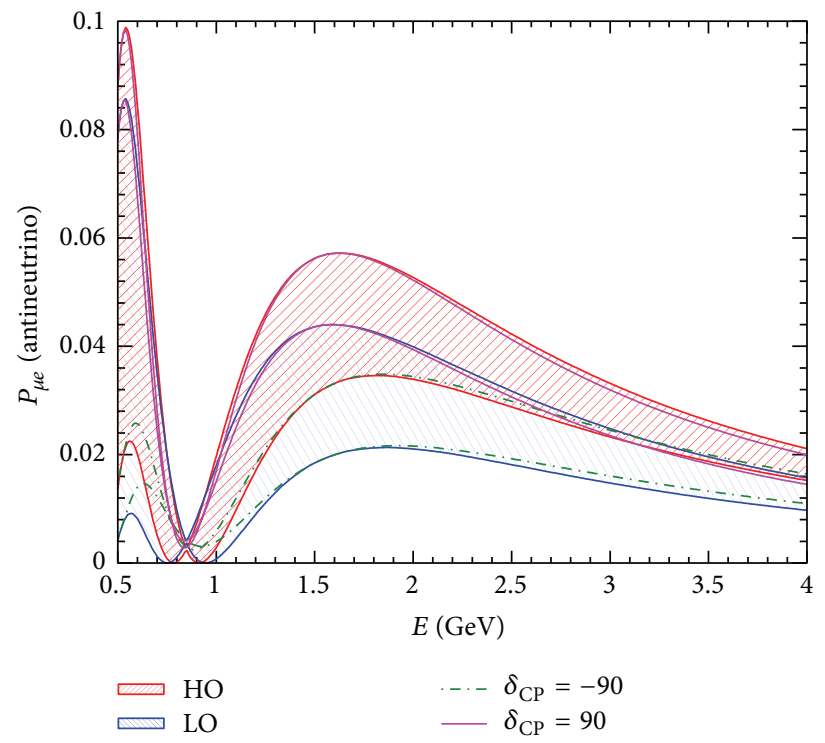

(d)

Figure 1: $P_{\mu e}$ energy spectrum for NO $v$ A experiment. The left (right) panel is for neutrino (antineutrino). The red (blue) band in the top panel corresponds to $\mathrm{NH}(\mathrm{IH})$, where we have used $\sin ^{2} \theta_{23}=0.5, \sin ^{2} 2 \theta_{13}=0.1$, and baseline $L=810 \mathrm{~km}$ and vary $\delta_{\mathrm{CP}}$ between $-\pi$ and $\pi$. The red (blue) band in the bottom panel is for HO (LO), where we have used $\sin ^{2} \theta_{23}=0.41(0.59)$ for LO (HO) and keep the hierarchy as normal. Inside each band the probability for $\delta_{\mathrm{CP}}=90^{\circ}\left(-90^{\circ}\right)$ case is shown by magenta (green) line.

expressions for long-baseline experiments $\mathrm{NO} v \mathrm{~A}, \mathrm{~T} 2 \mathrm{~K}$, and T2HK with negligible matter effects are given as

$$
\begin{aligned}
P_{\mu \mu}^{v}= & 1-\sin ^{2} 2 \theta_{23} \sin ^{2}\left[1.27 \frac{\Delta m_{31}^{2} L}{E}\right] \\
& +4 \sin ^{2} \theta_{13} \sin ^{2} \theta_{23} \cos 2 \theta_{23} \sin ^{2}\left[1.27 \frac{\Delta m_{31}^{2} L}{E}\right], \\
P_{\mu e}^{v}= & \sin ^{2} \theta_{23} \sin ^{2} 2 \theta_{13} \sin ^{2}\left[1.27 \frac{\Delta m_{31}^{2} L}{E}\right] .
\end{aligned}
$$

The leading order term in the $v_{\mu}$ survival probability $\left(P_{\mu \mu}^{v}\right)$ depends on $\sin ^{2} 2 \theta_{23}$ and one cannot distinguish between $P_{\mu \mu}^{v}\left(\theta_{23}\right)$ and $P_{\mu \mu}^{v}\left(\pi / 2-\theta_{23}\right)$. This kind of degeneracy that comes from the inherent structure of neutrino oscillation probability is called intrinsic octant degeneracy, whereas in the case of $P_{\mu e}^{v}$ the degeneracy of the octant with the parameter $\theta_{13}$ comes into play, since it depends on the parameter combination $\sin ^{2} \theta_{23} \sin ^{2} 2 \theta_{13}$. The values of $\theta_{23}$ in opposite octant for different values of $\theta_{13}$ and $\delta_{\mathrm{CP}}$ can have the same probabilities; that is, $P_{\mu e}^{v}\left(\theta_{23}, \theta_{13}, \delta_{\mathrm{CP}}\right)=P_{\mu e}^{v}(\pi / 2-$ $\left.\theta_{23}, \theta_{13}^{\prime}, \delta_{\mathrm{CP}}^{\prime}\right)$. This also gives rise to octant degeneracy. 

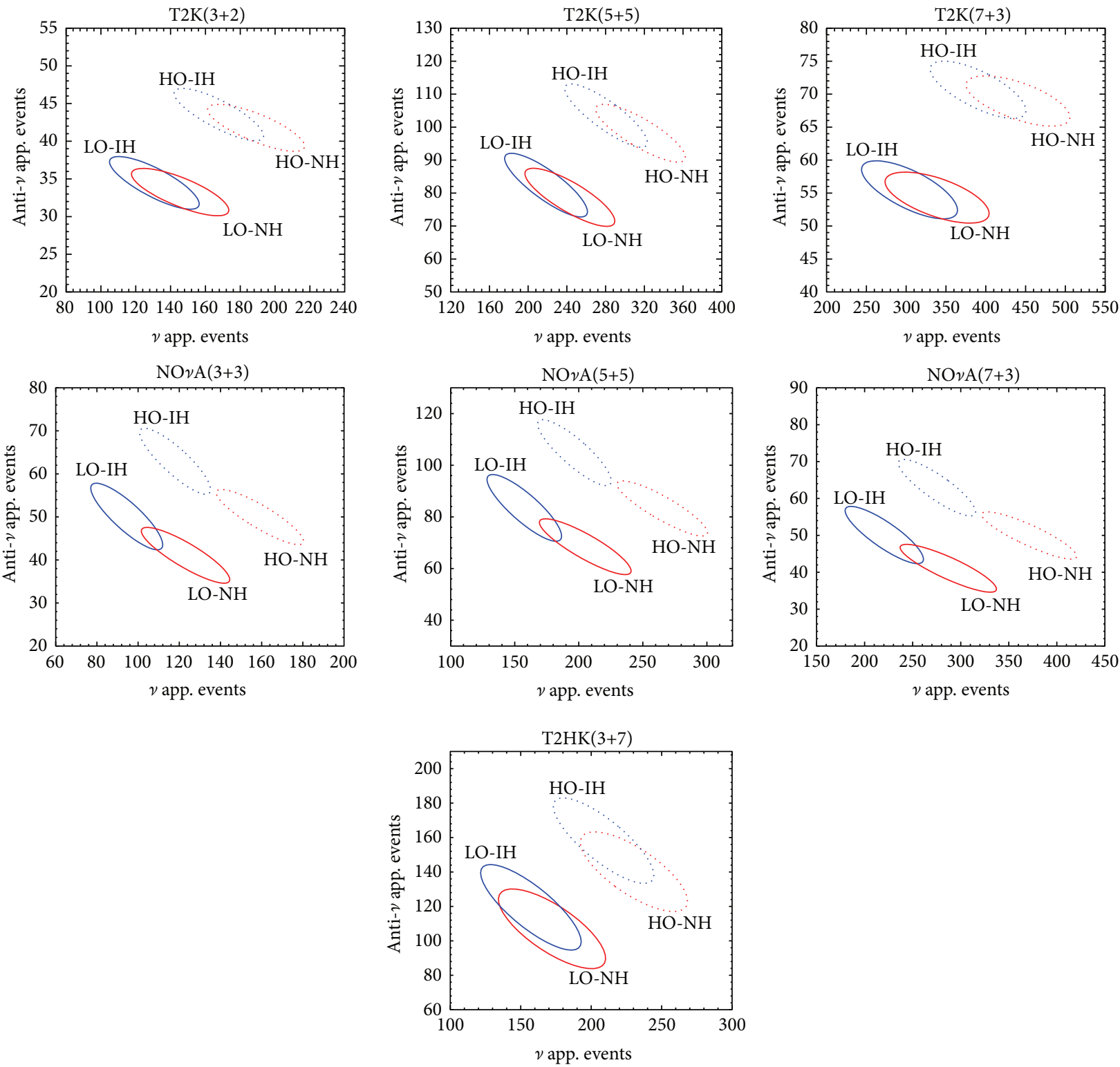

FIGURE 2: Neutrino and antineutrino appearance events for the $\nu_{\mu} \rightarrow \nu_{e}$ versus $\bar{\nu}_{\mu} \rightarrow \bar{\nu}_{e}$ channels by assuming both IH and NH and for lower and higher octants of $\theta_{23}$.

Before presenting the main results, we would like to discuss what one can expect about the determination of mass hierarchy and octant of $\theta_{23}$ from the biprobability plots, that is, neutrino-antineutrino appearance event rates. Figure 2 shows $v$ versus $\bar{v}$ events for all octant-hierarchy combinations. The blue curves are obtained by considering inverted hierarchy mass ordering and both of the values of $\theta_{23}$; that is, $\sin ^{2} \theta_{23}=0.41(\mathrm{LO})$ and $0.59(\mathrm{HO})$. The red curves are obtained by considering normal hierarchy mass ordering and $\mathrm{LO} / \mathrm{HO}$ values for $\sin ^{2} \theta_{23}$. These ellipses are plotted by obtaining event spectra for $(3+3)$ yrs, $(5+5)$ yrs, and $(7+3)$ yrs in $\nu$ and $\bar{v}$ mode for all values of $\delta_{\mathrm{CP}}$ for $\mathrm{NO} v \mathrm{~A}$, $\mathrm{T} 2 \mathrm{~K}$, and $(3+7)$ yrs for T2HK. Each point on $x$-axis $(y$-axis) represents the number of events measured by the respective experiments in neutrino (antineutrino) mode. The top panel represents the ellipses for $(3+2),(5+5)$, and $(7+3)$ years of running in neutrino and antineutrino modes for $\mathrm{T} 2 \mathrm{~K}$, the second panel represents the $\mathrm{NO} v \mathrm{~A}$ event rates for $(3+3)$, $(5+5)$, and $(7+3)$ years of run period, and the bottom panel represents the T2HK event rates for $(3+7)$ years of run period. For T2K and T2HK experiments the ellipses of both normal and inverted mass orderings overlap with each other for both the octants whereas for $\mathrm{NO} v \mathrm{~A}$ the overlap region is less (marginal) for LO (HO). Thus, it is very likely that the mass hierarchy and octant degeneracy could be probed better with the $\mathrm{NO} v \mathrm{~A}$ experiment.

Before doing the simulation, here we would like to emphasize that the relation between atmospheric parameters 

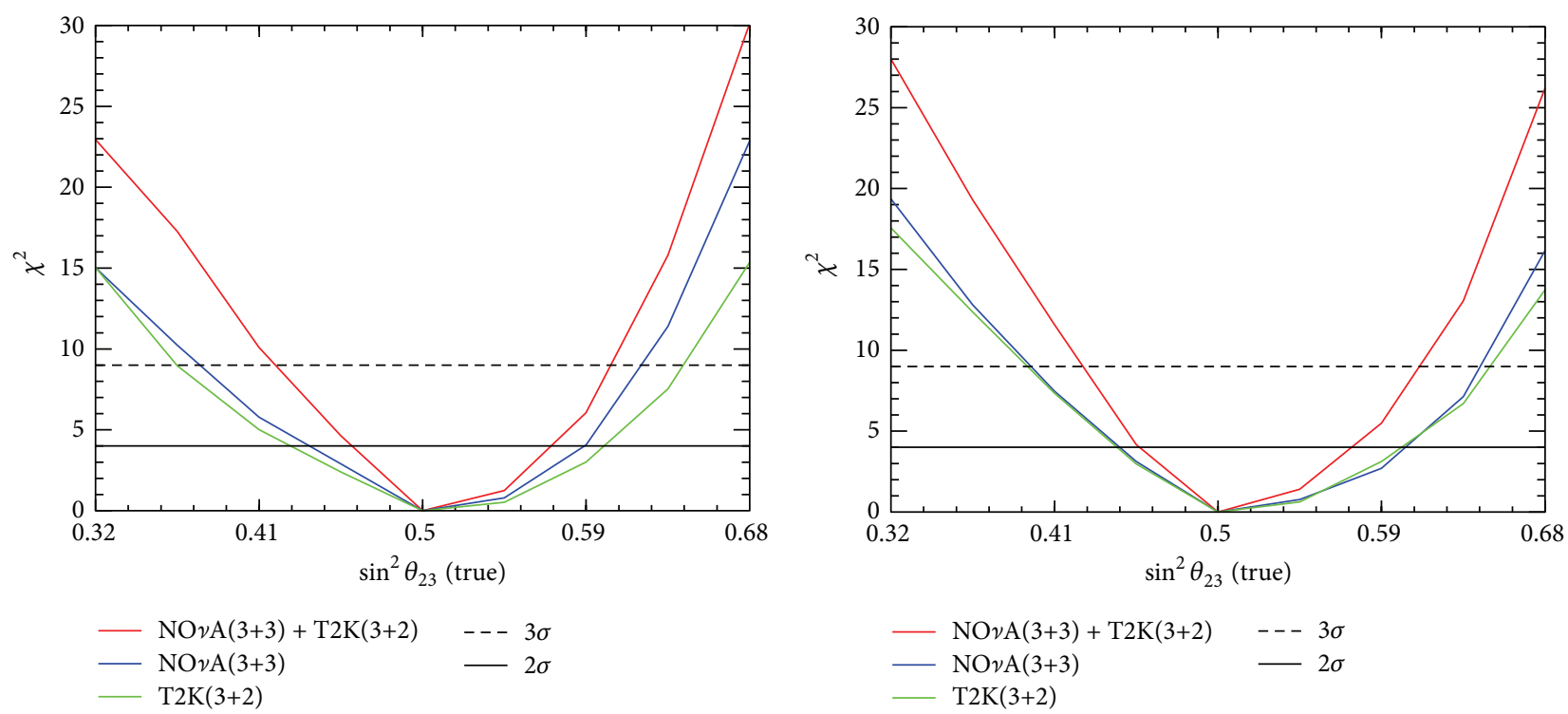

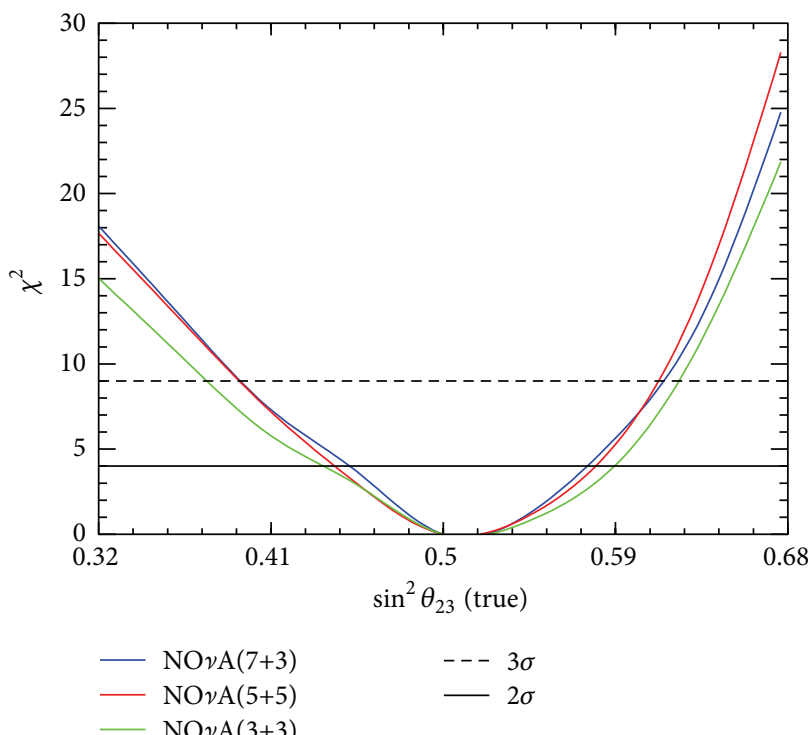

(a)

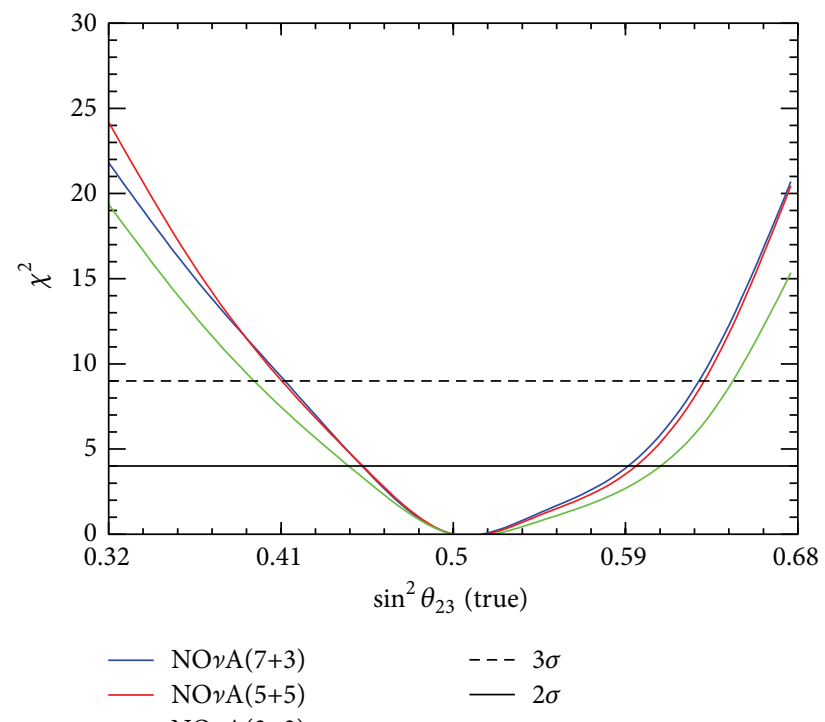

(b)

FIGURE 3: Octant sensitivity for a combination of T2K and NO $v$ A for the case of normal (left panel) and inverted (right panel) hierarchy.

$\left(\Delta m_{\mathrm{atm}}^{2}\right)$ and $\theta_{\mu \mu}$, measured in MINOS, and the standard oscillation parameters in nature are given as $[35,36]$

$$
\begin{aligned}
& \sin \theta_{23}=\frac{\sin \theta_{\mu \mu}}{\cos \theta_{13}}, \\
& \Delta m_{31}^{2} \\
& =\Delta m_{\mathrm{atm}}^{2} \\
& \quad+\left(\cos ^{2} \theta_{12}-\cos \delta_{\mathrm{CP}} \sin \theta_{13} \sin 2 \theta_{12} \tan \theta_{23}\right) \Delta m_{21}^{2} .
\end{aligned}
$$

It is clear from the above relations that the observed value of moderately large $\theta_{13}$ significantly affects the oscillation parameters. So here we use corrected definitions of these parameters to analyze octant sensitivity. We allocate measured values $\Delta m_{\mathrm{atm}}^{2}$ and $\theta_{\mu \mu}$ and calculate oscillation probabilities in terms of $\Delta m_{31}^{2}$ and $\theta_{23}$.

We consider the true values of oscillation parameters given in Table 3 and vary the test values of $\sin ^{2} \theta_{23}$ in LO (HO) for true higher octant (lower octant). We also marginalize over $\sin ^{2} 2 \theta_{13}$ in the range [0.07:0.13], $\delta_{\mathrm{CP}}$ in its full range, and $\Delta m_{\mathrm{atm}}^{2}$ in the range [2.05:2.75] $\times 10^{-3} \mathrm{eV}^{2}$ for $\mathrm{NH}$. The parameters $\theta_{12}$ and $\Delta m_{21}^{2}$ have been kept fixed in the analysis and priors for $\sin ^{2} 2 \theta_{13}$ and $\sin ^{2} \theta_{23}$ with $\sigma\left(\sin ^{2} 2 \theta_{13}\right)=0.01$ and $\sigma\left(\sin ^{2} \theta_{23}\right)=0.05$ are also added.

We simulate the long-baseline experiments $\mathrm{T} 2 \mathrm{~K}$ and $\mathrm{NO} v \mathrm{~A}$ using the GLoBES package. For T2K, we assume 3 years of running in neutrino mode and 2 years in antineutrino 

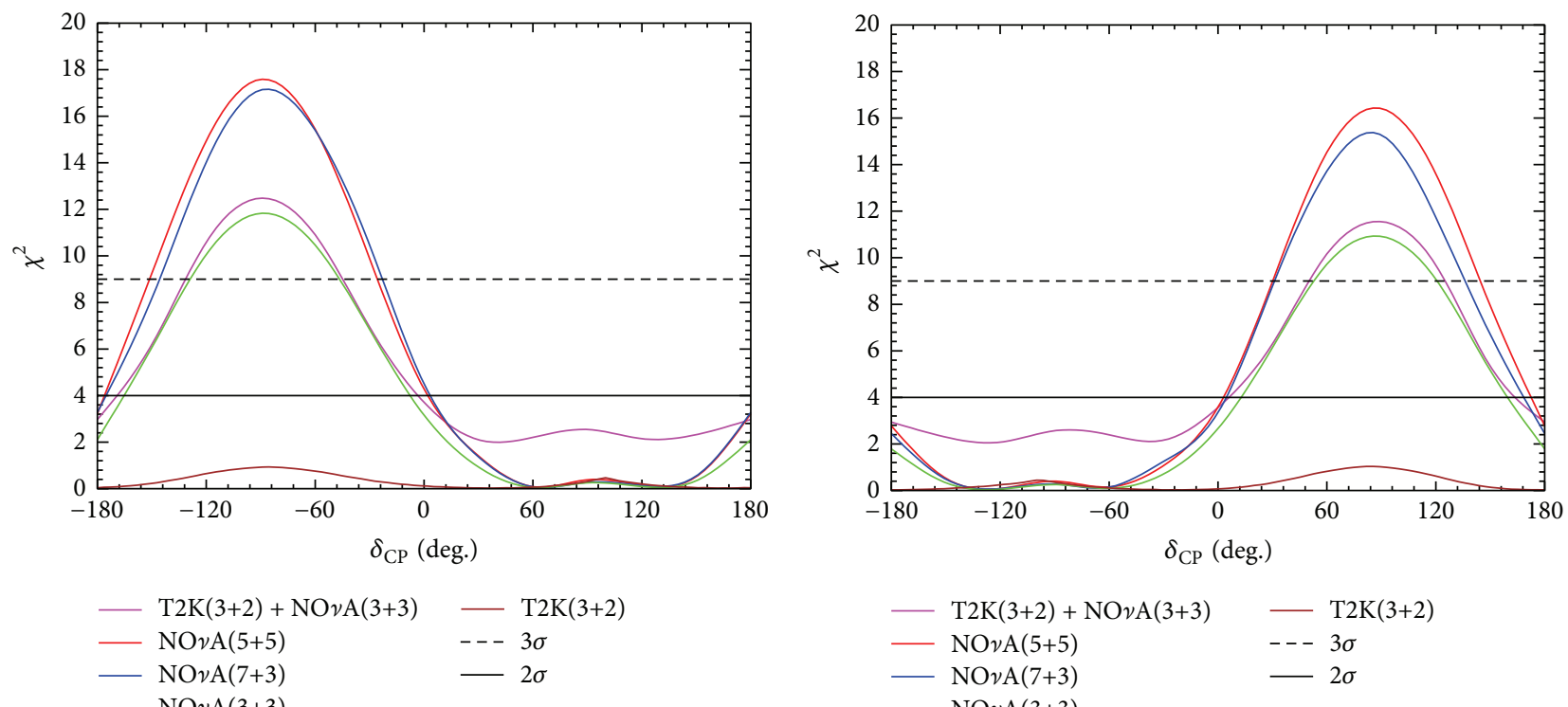

$-\mathrm{NO} v \mathrm{~A}(3+3)$

$-\mathrm{NO} v \mathrm{~A}(3+3)$
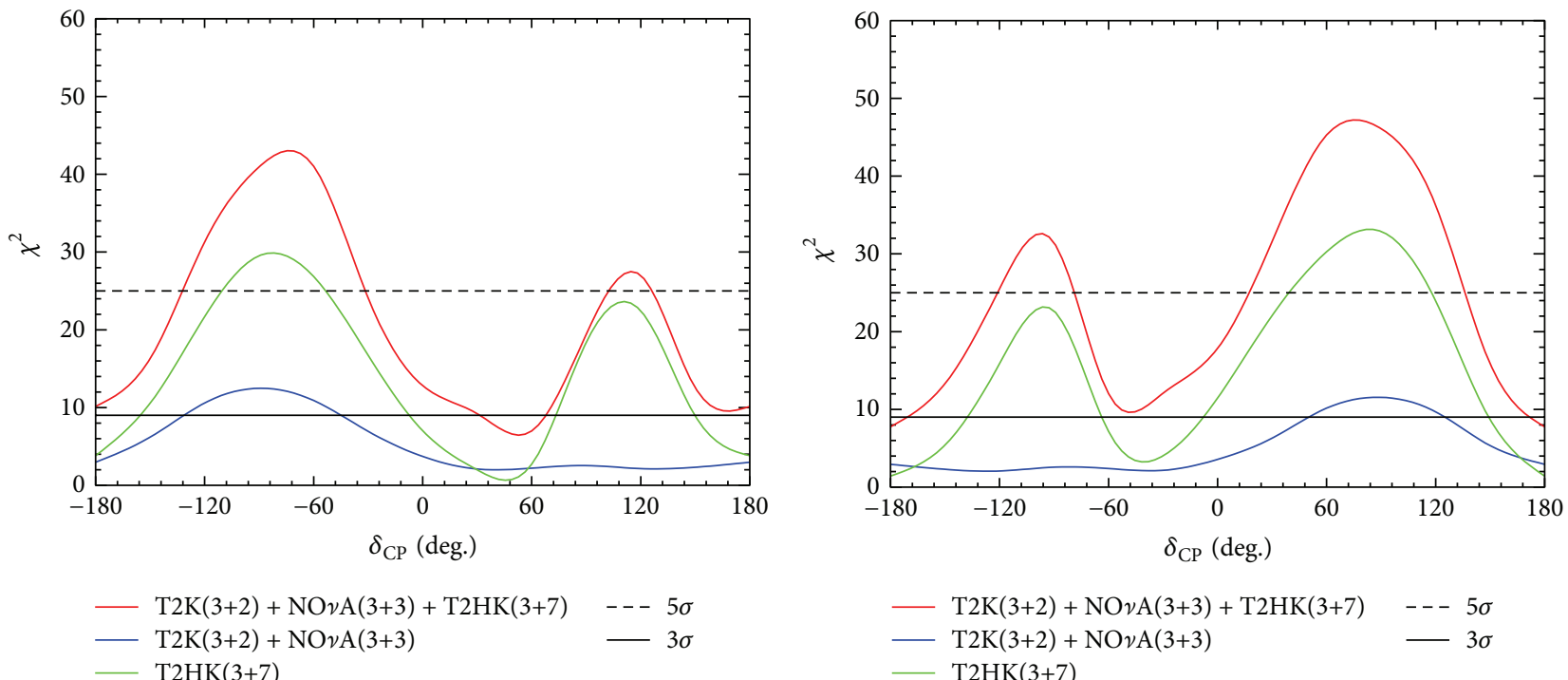

(a)

(b)

FiguRE 4: Mass hierarchy significance as a function of true $\delta_{\mathrm{CP}}$. In the left panel normal hierarchy is considered as true hierarchy and inverted is taken as test hierarchy and in the right panel inverted hierarchy is considered true hierarchy and normal is taken as test hierarchy.

mode and for $\mathrm{NO} v \mathrm{~A}$, we consider 3 years of neutrino running followed by 3 years of antineutrino running. Furthermore, we also consider the case if $\mathrm{NO} v \mathrm{~A}$ continues the data taking for ten years beyond its scheduled $(3+3)$ years and perform the analysis for two possible combinations $(5+5)$ and $(7+3)$ years of running.

In Figure 3, we illustrate the ability of $\mathrm{NO} v \mathrm{~A}$ experiment to determine the octant as a function of the true value of $\theta_{23}$. The values of $\chi^{2}$ are evaluated using the standard rules as described in GLoBES. The green, red, and blue curves (in the bottom panel) represent the octant resolution of $\mathrm{NO} v \mathrm{~A}$ with $(3+3),(5+5)$, and $(7+3)$ yrs of runs in $v$ and $\bar{v}$ modes, respectively. From Figure 3, it can be seen that, with only T2K data of $(3+2)$ years of run, it is possible to resolve the octant degeneracy with $2 \sigma$ significance if the true $\sin ^{2} \theta_{23}$ will lie around $0.41(\mathrm{LO})$ or $0.59(\mathrm{HO})$ and one can have a better sensitivity for $\mathrm{NO} v \mathrm{~A}$ experiment with $(3+3)$ yrs of run period. The significance increases significantly if we combine the data from both $\mathrm{T} 2 \mathrm{~K}$ and $\mathrm{NO} v \mathrm{~A}$ as seen from the top panels. For ten years of $\mathrm{NO} v \mathrm{~A}$ run, although we get a better sensitivity than that of $(3+3)$ yrs of run, there is no significant difference between $(5+5)$ yrs and $(7+3)$ years of running.

\section{Mass Hierarchy Determination}

Determination of neutrino mass hierarchy is one of the outstanding issues in neutrino oscillation physics. The conventional method to achieve this is by using matter effects 

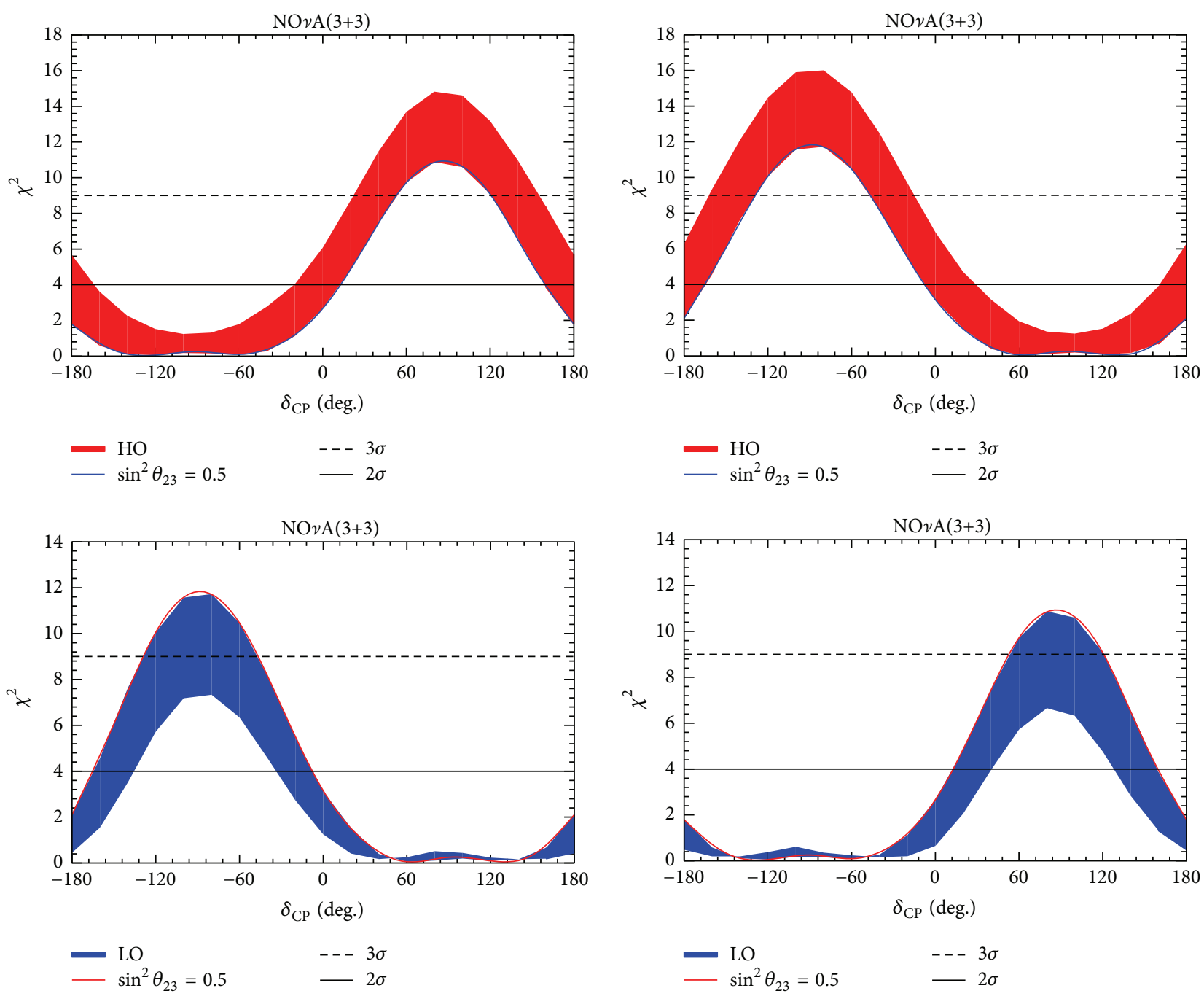

FIgURE 5: Mass hierarchy significance with the octant of $\theta_{23}$ for the scheduled run of NO $v$ A experiment.

in very long-baseline neutrino oscillation experiments, as the matter effects enhance the separation between oscillation spectra and, therefore, the event spectra between the normal and inverted hierarchy. In this section we describe the capabilities of $\mathrm{T} 2 \mathrm{~K}, \mathrm{NO} \nu \mathrm{A}$, and $\mathrm{T} 2 \mathrm{HK}$ experiments for the determination of mass hierarchy.

The value of $\chi^{2}$ has been obtained by using the true parameters as listed in Table 3 , except that of $\sin ^{2} \theta_{23}$, which is taken to be 0.5 . The true value of $\delta_{\mathrm{CP}}$ is varied within its full range, that is, in $[-\pi, \pi]$, and test value of $\Delta m_{\mathrm{atm}}^{2}$ is varied in $\mathrm{IH}(\mathrm{NH})$ range for true $\mathrm{NH}(\mathrm{IH})$. We also marginalize over $\sin ^{2} 2 \theta_{13}$ and $\sin ^{2} \theta_{23}$ in their $3 \sigma$ ranges and add prior to $\sin ^{2} 2 \theta_{13}$ with $\sigma\left(\sin ^{2} 2 \theta_{13}\right)=0.01$.

In Figure 4, we present the hierarchy determination sensitivity of $\mathrm{T} 2 \mathrm{~K}, \mathrm{NO} \nu \mathrm{A}$, and $\mathrm{T} 2 \mathrm{HK}$ as a function of true value of $\delta_{\mathrm{CP}}$. We assume $\mathrm{NH}(\mathrm{IH})$ to be the true hierarchy in the left (right) panel. It can be seen that the wrong hierarchy can be ruled out quite effectively in the LHP (UHP) for $\mathrm{NH}$ (IH), which is basically the favorable half plane and in the other half plane the mass hierarchy cannot be determined effectively for $\mathrm{T} 2 \mathrm{~K}$ and $\mathrm{NO} \nu \mathrm{A}$ experiments. However, the combined data from these two experiments $(\mathrm{T} 2 \mathrm{~K}(3+2)$ and $\mathrm{NO} v \mathrm{~A}(3+3))$ improves the situation significantly and the sensitivity increases to more than $1 \sigma$ for all values of $\delta_{\mathrm{CP}}$. The mass hierarchy significance above $3 \sigma$ has a $\delta_{\mathrm{CP}}$ coverage of $75 \%$ for $\mathrm{T} 2 \mathrm{HK}$ experiment alone and $90 \%$ for combined data of $\mathrm{T} 2 \mathrm{~K}, \mathrm{NO} \nu \mathrm{A}$, and $\mathrm{T} 2 \mathrm{HK}$ experiments.

Next, we would like to study the effect of $\theta_{23}$ octant on the $\mathrm{MH}$ sensitivity. We obtain the $\mathrm{MH}$ sensitivity by varying the true value of $\sin ^{2} \theta_{23}$ in $\mathrm{LO}(\mathrm{HO})$ which has been shown in Figure 5 where the red (blue) band in the top (bottom) panel corresponds to $\mathrm{HO}(\mathrm{LO})$. It is clear from the figure that the $\mathrm{MH}$ sensitivity is significantly large if the value of $\sin ^{2} \theta_{23}$ is in higher octant.

\section{CP Violation Discovery Potential}

Accelerator based long-baseline neutrino oscillation experiments can address CP violation problem through the appearance channels of $\nu_{\mu} \rightarrow \nu_{e}$ and $\bar{\nu}_{\mu} \rightarrow \bar{\nu}_{e}$. From (1) we can see 

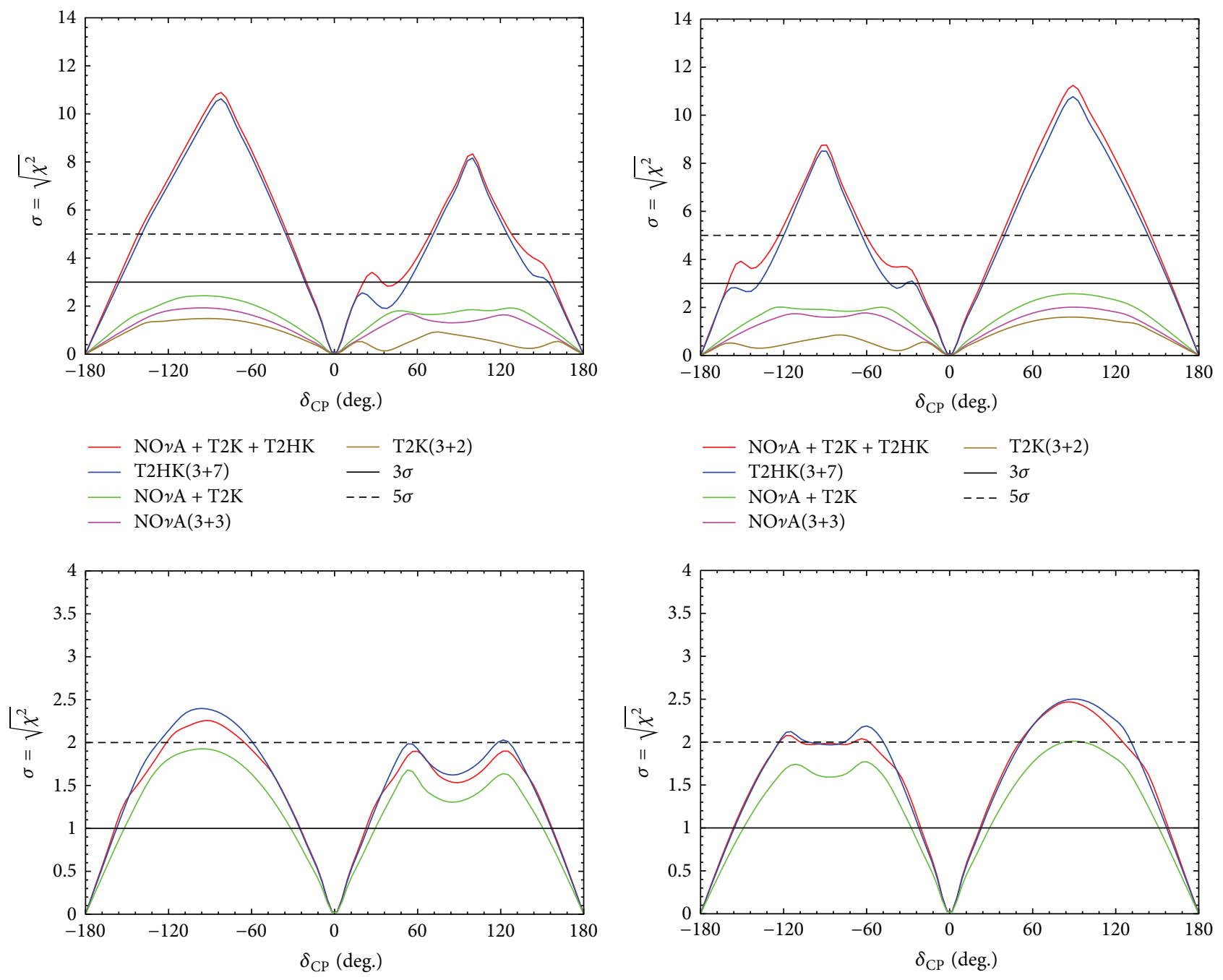

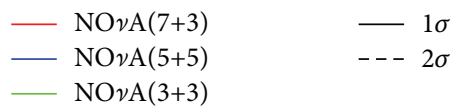

(a)

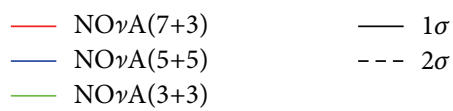

(b)

FIGURE 6: CP violation sensitivity for different combinations of run time of T2K, NO $v$ A, and T2HK experiment for $\mathrm{NH}$ (IH) in the left (right) panel.

that the $\mathrm{CP}$ violating effects due to $\delta_{\mathrm{CP}}$ are modified by all the three mixing angles and their combinations, thus resulting in an eightfold parameter degeneracy. In order to obtain the significance of $\mathrm{CP}$ violation sensitivity, we simulate the true event spectrum by keeping the true values of oscillation parameters as in Table 3 except for $\sin ^{2} \theta_{23}=0.5$ and vary the true value of $\delta_{\mathrm{CP}}$ in the range $[-\pi, \pi]$. We then compare those with test event spectrum for $\delta_{\mathrm{CP}}=0$ or $\pi$ and, thus, obtain the minimum $\chi^{2}$. We consider the sign degeneracy of $\Delta m_{31}^{2}$ by marginalizing over it, in both $\mathrm{NH}$ and $\mathrm{IH} 3 \sigma$ ranges. We also do marginalization over $\sin ^{2} 2 \theta_{13}$ and $\sin ^{2} \theta_{23}$ in their $3 \sigma$ and added prior for $\sin ^{2} 2 \theta_{13}$.

In Figure 6, we plot the sensitivity to rule out the CP conserving scenarios, as a function of true $\delta_{\mathrm{CP}}$ assuming $\mathrm{NH}$ (IH) to be the true hierarchy in the left panel (right panel).
From the figure one can notice that T2K by itself has no $\mathrm{CP}$ violation sensitivity at $2 \sigma \mathrm{CL}$. For $\mathrm{NO} \nu \mathrm{A}$ with $(3+3)$ years of running, there will be $\mathrm{CP}$ violation sensitivity above $1.5 \sigma$ level for about one-third of the CP violating phase $\delta_{\mathrm{CP}}$ space. Furthermore, the synergistic combination of $\mathrm{NO} v \mathrm{~A}$ and $\mathrm{T} 2 \mathrm{~K}$ leads to much better $\mathrm{CP}$ violation sensitivity compared to the individual capabilities. Even the combination of $\mathrm{NO} v \mathrm{~A}(3+3)$ and $\mathrm{T} 2 \mathrm{~K}(3+2)$ has comparable sensitivity as for 10 -year running of $\mathrm{NO} v \mathrm{~A}$. Owing to the fact that main goal of T2HK experiment is to determine $\mathrm{CP}$ violation, one can see that it has a significance of above $5 \sigma \mathrm{CL}$ for a fraction of two-fifths values of the CP violating phase $\delta_{\mathrm{CP}}$ space. This in turn boosts up the sensitivity when its data is added to $\mathrm{NO} v \mathrm{~A}(3+3) \mathrm{yrs}$ and T2K $(3+2)$ yrs. From the plots in the lower panel, one can observe that the sensitivity of $\mathrm{NO} v \mathrm{~A}$ increases slightly for 10 


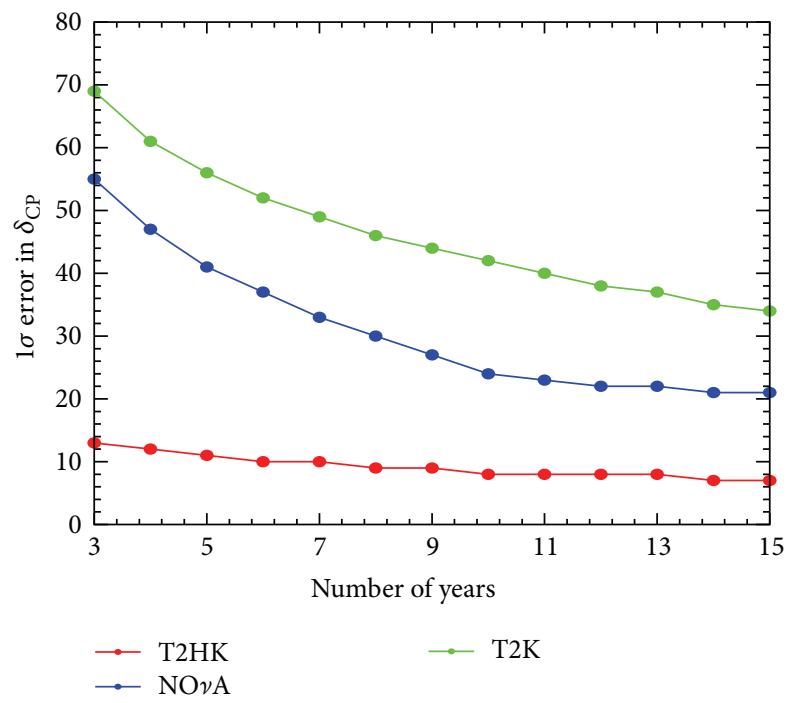

(a)

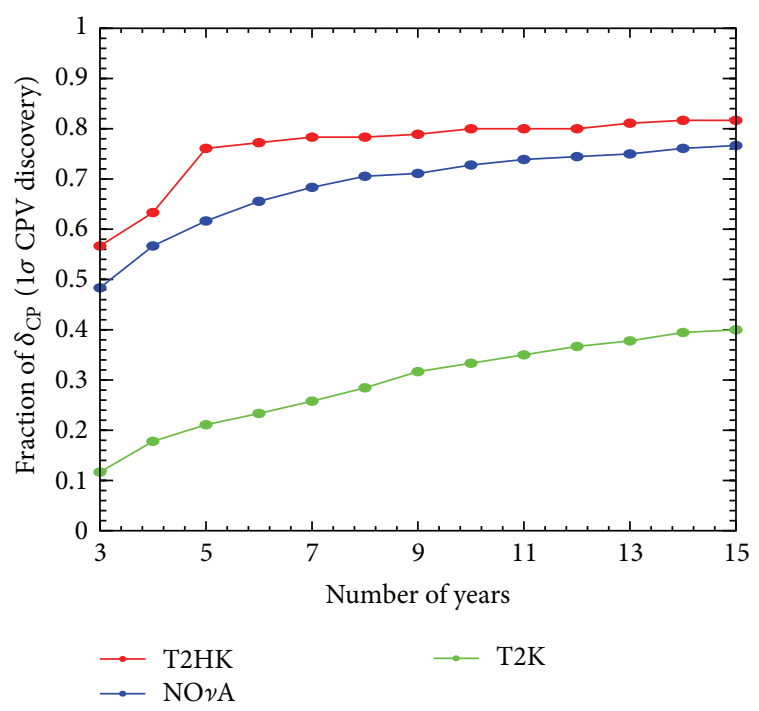

(b)

Figure 7: Sensitivity versus running time: $1 \sigma$ error in $\delta_{\mathrm{CP}}$ as a function of running time in years for true value of $\delta_{\mathrm{CP}}=0$ (left panel). The fraction of $\delta_{\mathrm{CP}}$ for which $\delta_{\mathrm{CP}}=0^{\circ}, 180^{\circ}$ is excluded with $1 \sigma$ as a function of running time.

years of run time, with $(5 v+5 \bar{\nu})$ combination having better sensitivity than that of $(7 v+3 \bar{\nu})$ combination. From the figure, we can see that there is drop in the $\chi^{2}$ values in the half planes of $\delta_{\mathrm{CP}}$, that is, in the region $[0,180]^{\circ}\left([-180,0]^{\circ}\right)$ for $\mathrm{NH}(\mathrm{IH})$. Marginalization over both the hierarchies causes this drop in the CPV sensitivity for unfavorable values of $\delta_{\mathrm{CP}}$.

In Figure 7, the left panel shows the $1 \sigma$ uncertainty of $\delta_{\mathrm{CP}}$ as a function of running time (in years) for true value of $\delta_{\mathrm{CP}}=0$ and the right panel shows the $\mathrm{CP}$ violation sensitivity as a function of running time. In both cases, the ratio of neutrino and antineutrino modes is fixed to $1: 1$ for T2K and $\mathrm{NO} v \mathrm{~A}$ and $3: 7$ for T2HK. In this analysis, mass hierarchy is assumed to be unknown. Therefore, we have marginalized over both the hierarchies, and the result shown in Figure 7 is for the true hierarchy as normal hierarchy. From the left panel of the figure, we can see that the values of $\delta_{\mathrm{CP}}$ can be determined to be better than $35^{\circ}\left(21^{\circ}\right)$ for all values of $\delta_{\mathrm{CP}}$ for $\mathrm{T} 2 \mathrm{~K}(\mathrm{NO} v \mathrm{~A})$. In the case of T2HK the values of $\delta_{\mathrm{CP}}$ can be determined to be better than $9^{\circ}$ for all values of $\delta_{C P}$. From the right panel, we can see that $\mathrm{CP}$ violation can be observed with more than $1 \sigma$ significance for $40(75) \%$ of the possible values of $\delta_{\mathrm{CP}}$ for T2K (NO $\left.v \mathrm{~A}\right)$, whereas, for T2HK, CP violation can be observed with more than $1 \sigma$ significance for $80 \%$ of the possible values of $\delta_{\mathrm{CP}}$.

5.1. Correlation between $\delta_{\mathrm{CP}}$ and $\theta_{13}$. The knowledge of reactor mixing angle $\theta_{13}$ plays a crucial role in the discovery potential of $\delta_{\mathrm{CP}}$. The recent discovery of large value of $\theta_{13}$ has established the need to study and understand the dependency between $\delta_{\mathrm{CP}}$ and $\theta_{13}$. In this subsection, we discuss the correlation between the oscillation parameters $\theta_{13}$ and $\delta_{\mathrm{CP}}$. To obtain the confidence region, we have fixed the true values as in Table 3 and considered true $\sin ^{2} \theta_{23}=0.59$ for higher octant and true $\sin ^{2} \theta_{23}=0.41$ for lower octant, since the octant of $\theta_{23}$ is not known. We have varied the test value of $\sin ^{2} 2 \theta_{13}$ in its $3 \sigma$ range. In this analysis we have kept both true and test hierarchy as normal hierarchy.

Figure 8 shows the confidence regions in the $\sin ^{2} 2 \theta_{13}-\delta_{C P}$ plane for different combinations of $\mathrm{T} 2 \mathrm{~K}$ and $\mathrm{NO} v \mathrm{~A}$ experiments. One can see from these figures that at $2 \sigma$ confidence level, the uncertainty in the knowledge of $\theta_{23}$ octant has a noticeable effect on the correlation between $\delta_{\mathrm{CP}}$ and $\theta_{13}$.

5.2. Correlation between $\delta_{\mathrm{CP}}$ and $\theta_{23}$. From the previous subsection, we can see that the uncertainty in $\theta_{23}$ has a very large impact on determination of neutrino oscillation parameters. Thus, it is important to understand the exclusive correlation between $\delta_{\mathrm{CP}}$ and $\theta_{23}$ while keeping the true values of rest of the oscillation parameters fixed. In this subsection, we discuss the correlation between the oscillation parameters $\theta_{23}$ and $\delta_{\mathrm{CP}}$. For our analysis, we have kept true values of oscillation parameters as in Table 3. We vary the test values of $\sin ^{2} \theta_{23}$ and $\delta_{\mathrm{CP}}$ in their $3 \sigma$ ranges. The obtained results of the confidence regions in $\sin ^{2} \theta_{23}-\delta_{\mathrm{CP}}$ plane are shown in Figures 9 and 10 for different combinations of T2K and NOA experiments.

\section{Summary and Conclusion}

With the recent discovery of the last unknown reactor mixing angle $\theta_{13}$, the mechanism of three-flavor neutrino mixing pattern is now well established. But still there are several issues related to neutrino oscillation parameters that remain open, namely, the absolute mass scale of neutrinos, determination of the mass hierarchy, octant of the atmospheric mixing angle $\theta_{23}$, the magnitude of the $\mathrm{CP}$ violating phase $\delta_{\mathrm{CP}}$, and the observation of $\mathrm{CP}$ violation in the neutrino sector. Therefore, the main focus of the current and future 

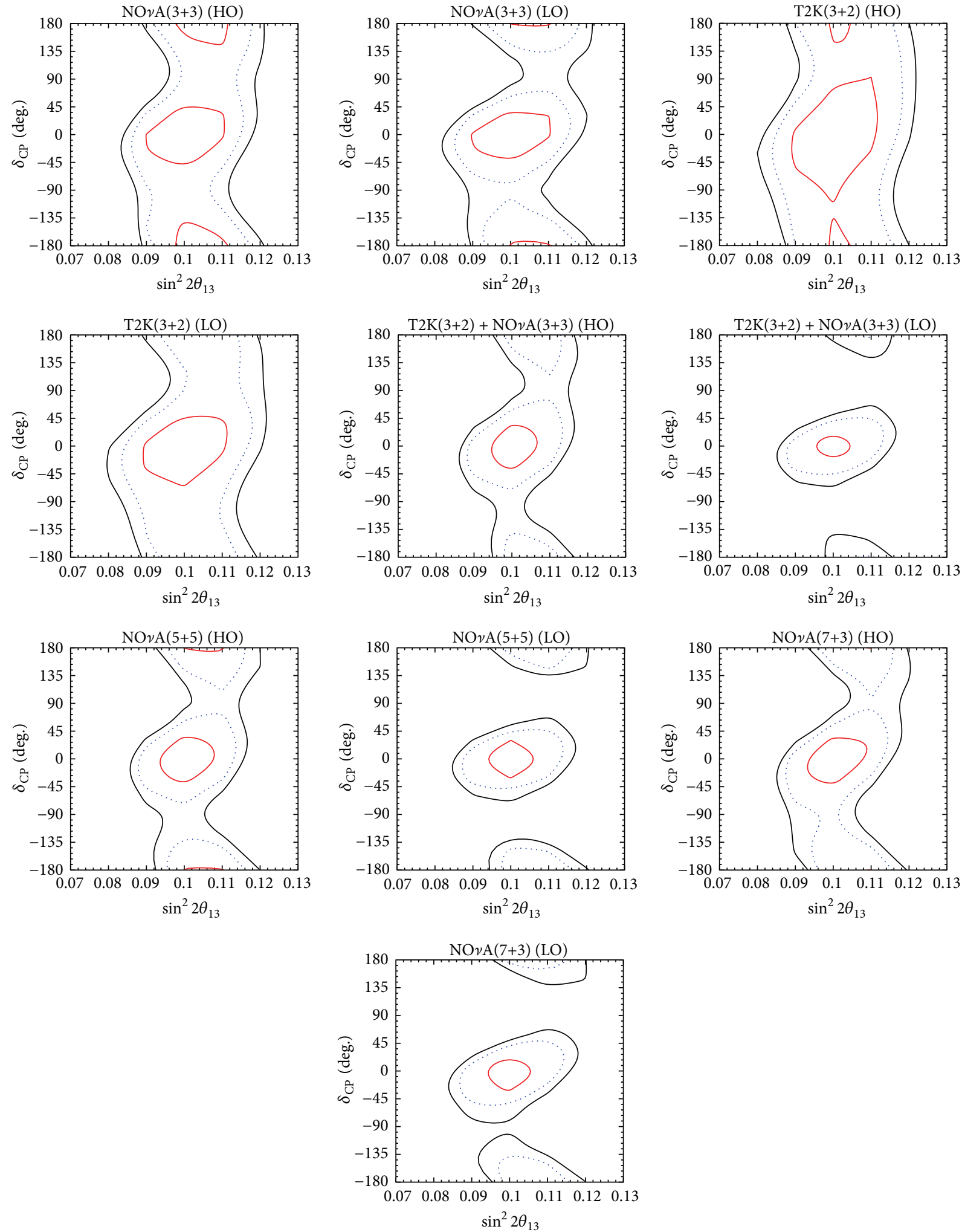

FIGURE 8: Confidence region in $\sin ^{2} 2 \theta_{13}-\delta_{\mathrm{CP}}$ plane for $\delta_{\mathrm{CP}}=0$ and for different run combinations of T2K and NO $\nu \mathrm{A}$ experiments, where the red, blue, and black contours represent the $1 \sigma(68.3 \% \mathrm{CL}), 1.64 \sigma(90 \% \mathrm{CL})$, and $2 \sigma(95.45 \% \mathrm{CL})$ values, respectively, for two degrees of freedom. 

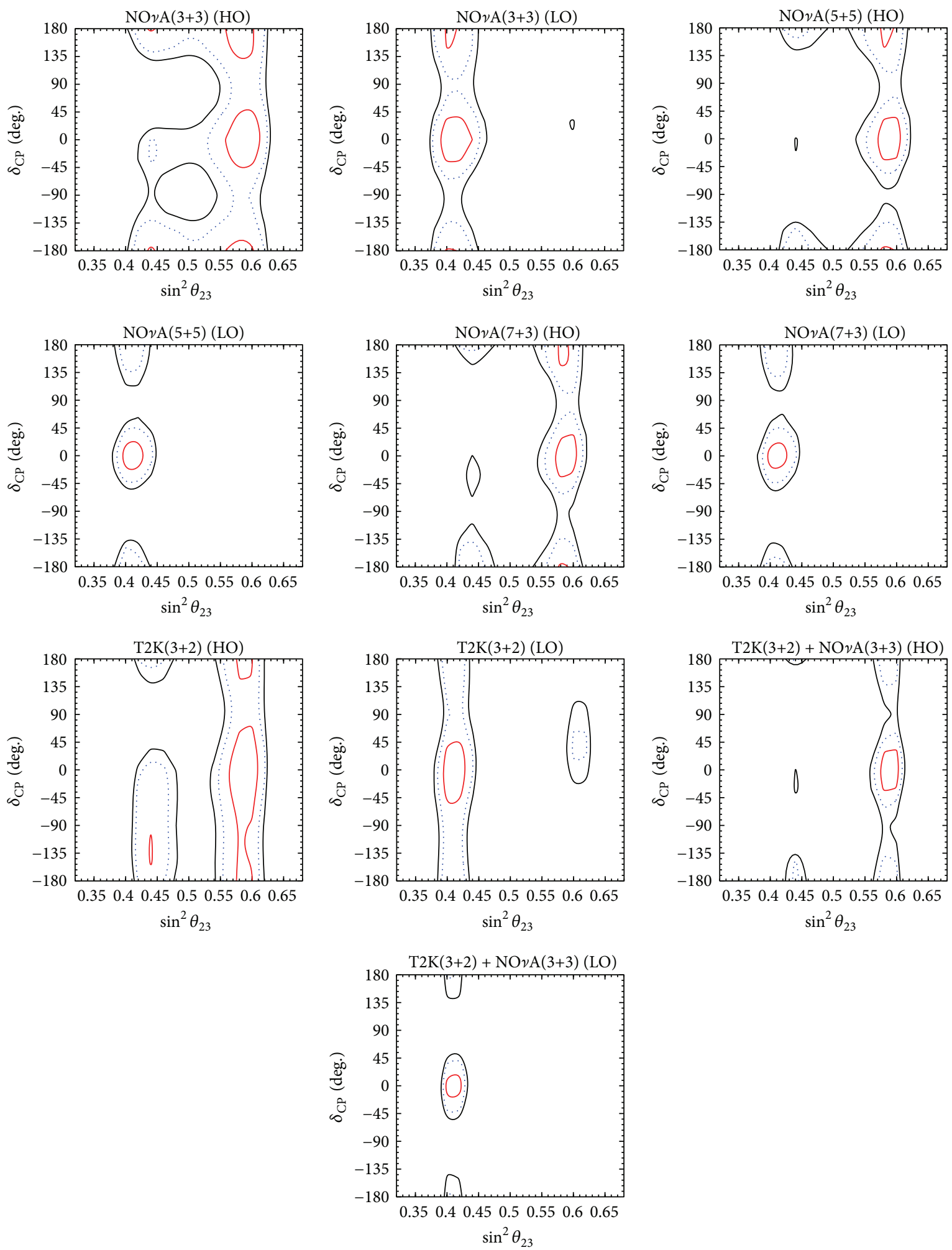

FIGURE 9: Confidence region in $\sin ^{2} \theta_{23}-\delta_{\mathrm{CP}}$ plane for true $\delta_{\mathrm{CP}}=0$, where red, blue, and black contours represent the $1 \sigma(68.3 \% \mathrm{CL}), 1.64 \sigma$ $(90 \% \mathrm{CL})$, and $2 \sigma(95.45 \% \mathrm{CL})$ values, respectively, for two degrees of freedom. Here hierarchy is assumed to be IH. 

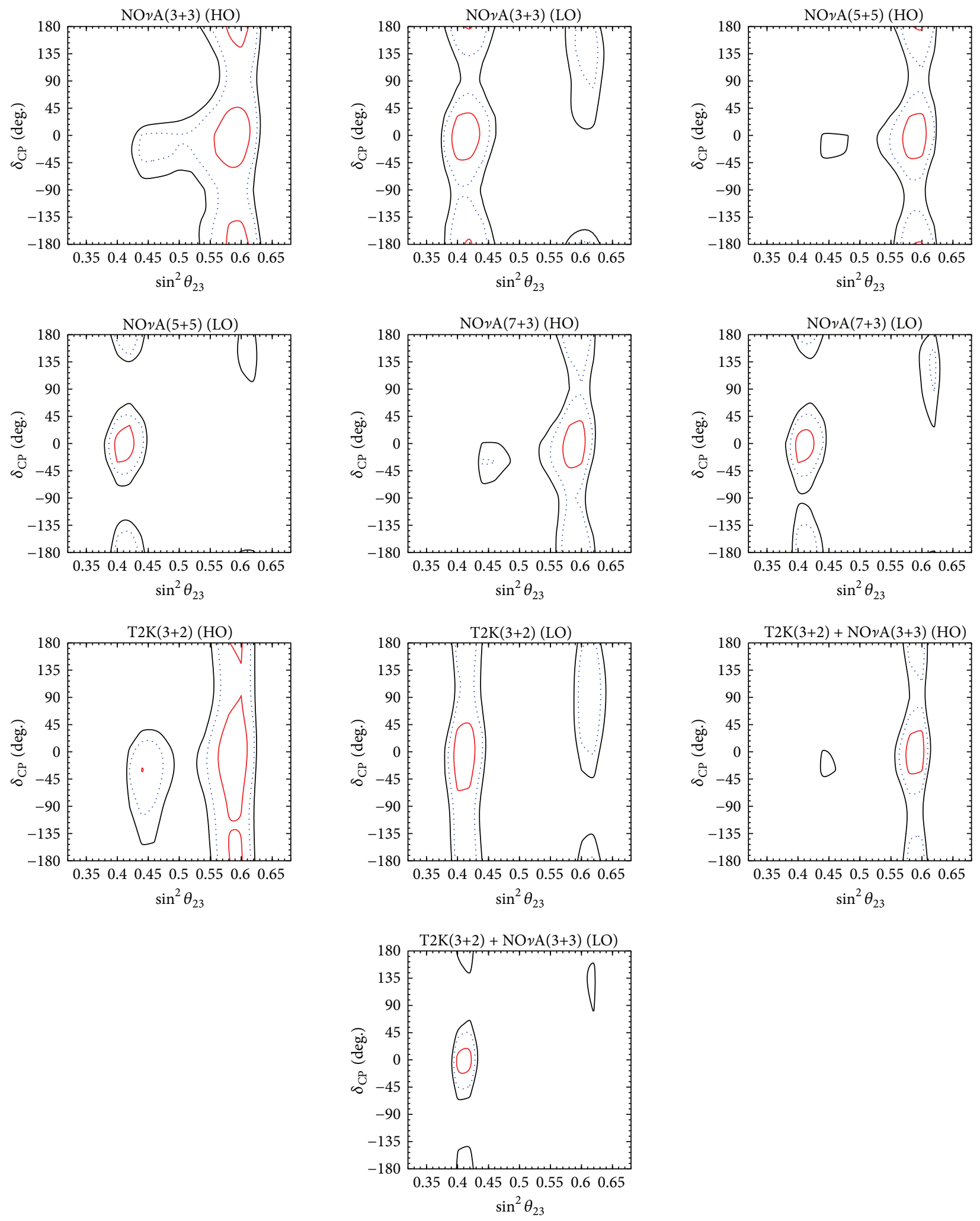

FIGURE 10: Confidence region in $\sin ^{2} \theta_{23}-\delta_{\mathrm{CP}}$ plane for true $\delta_{\mathrm{CP}}=0$, where red, blue, and black contours represent the $1 \sigma$, $1.64 \sigma$, and $2 \sigma$ values, respectively, for two degrees of freedom. Here hierarchy is assumed to be $\mathrm{NH}$. 
oscillation experiments is to provide answers to some of these unsolved questions.

In this paper, we have investigated the prospects of the determination of mass hierarchy, the octant of $\theta_{23}$, and the observation of $\mathrm{CP}$ violation in the neutrino sector due to $\delta_{\mathrm{CP}}$ with the currently running accelerator based neutrino experiments $\mathrm{NO} v \mathrm{~A}$ and $\mathrm{T} 2 \mathrm{~K}$ and the forthcoming $\mathrm{T} 2 \mathrm{HK}$ experiment. As the reactor mixing angle $\theta_{13}$ is now known to be significantly large, the oscillation probability $P\left(v_{\mu} \rightarrow v_{e}\right)$ and its corresponding antineutrino counterpart are sensitive for the determination of mass hierarchy and $\theta_{23}$ octant. We found that T2K experiment with $(3 v+2 \bar{v})$ years of running can resolve the octant degeneracy with nearly $2 \sigma \mathrm{CL}$ if the true value of $\theta_{23}$ is around $\sin ^{2} \theta_{23}=0.41$ (LO) or $\sin ^{2} \theta_{23}=$ 0.59 (HO). The sensitivity increases to nearly $3 \sigma$ with $(3 v+$ $3 \bar{v})$ years of running of $\mathrm{NO} v \mathrm{~A}$. However, if we combine the data from these two experiments the sensitivity increases significantly than the sensitivities of individual experiments. Furthermore, if we assume that $\mathrm{NO} v \mathrm{~A}$ continues data taking for 10 years then octant degeneracy can be resolved with $\mathrm{NO} \nu \mathrm{A}$ experiment alone with more than $3 \sigma$ significance. For the determination of mass hierarchy, it is also possible to rule out nearly one-third of the $\delta_{\mathrm{CP}}$ space at $3 \sigma \mathrm{CL}$ if we use the synergy between $\mathrm{NO} v \mathrm{~A}$ and $\mathrm{T} 2 \mathrm{~K}$ experiments. In this case the sensitivity increases significantly for ten years of running of $\mathrm{NO} v \mathrm{~A}$ with $(5+5)$ combination that is found to be more suitable than the combination of $(7+3)$ years.

Measuring CP violation in the lepton sector is another important challenging problem today. We have also performed a systematic study of the CP sensitivity of the current long-baseline experiments $\mathrm{T} 2 \mathrm{~K}$ and $\mathrm{NO} v \mathrm{~A}$. Although these experiments are not planned to study leptonic $\mathrm{CP}$ violation, we analyze the synergies between these setups which may aid in $\mathrm{CP}$ violation discovery by constraining the value of $\delta_{\mathrm{CP}}$. Although dedicated long-baseline experiments like DUNE, T2HK are planned to study CP violation in neutrino sector, we may have the first hand information on $\delta_{\mathrm{CP}}$ from these experiments much before those dedicated facilities are operational. We found that $\mathrm{T} 2 \mathrm{~K}$ by itself has marginal $\mathrm{CP}$ violation sensitivity at $1 \sigma \mathrm{CL}$. For $\mathrm{NO} \nu \mathrm{A}$ with $(3+3)$ years of running there will be $\mathrm{CP}$ violation sensitivity above $1.5 \sigma$ level for about one-third of the $\mathrm{CP}$ violating phase $\delta_{\mathrm{CP}}$ space. The sensitivity increases slightly for 10 years of run time, with $(5 v+5 \bar{v})$ combination having sensitivity compared to that of $(7 v+3 \bar{v})$ combination. The data from T2HK experiment will improve the CPV sensitivity significantly. We have also found that the CP violating phase $\delta_{\mathrm{CP}}$ can be determined to be better than $35^{\circ}, 21^{\circ}$, and $9^{\circ}$ for all values of $\delta_{\mathrm{CP}}$ for $\mathrm{T} 2 \mathrm{~K}, \mathrm{NO} v \mathrm{~A}$, and $\mathrm{T} 2 \mathrm{HK}$ experiments. We also obtained the confidence regions in the $\delta_{\mathrm{CP}}-\theta_{13}\left(\theta_{23}\right)$ plane for both T2K and $\mathrm{NO} v \mathrm{~A}$ experiments.

\section{Conflict of Interests}

The authors declare that there is no conflict of interests regarding the publication of this paper.

\section{Acknowledgments}

C. Soumya and K. N. Deepthi would like to thank University Grants Commission for financial support. The work of R. Mohanta was partly supported by the Council of Scientific and Industrial Research, Government of India, through Grant no. 03(1190)/11/EMR-II. The authors would like to thank Drs. S. K. Agarwalla and S. K. Raut for many useful discussions regarding GLoBES.

\section{References}

[1] B. Pontecorvo, "Inverse beta processes and nonconservation of lepton charge," Soviet Physics-JETP, vol. 7, pp. 172-173, 1958.

[2] Z. Maki, M. Nakagawa, and S. Sakata, "Remarks on the unified model of elementary particles," Progress of Theoretical Physics, vol. 28, no. 5, pp. 870-880, 1962.

[3] F. P. An, J. Z. Bai, A. B. Balantekin et al., "Observation of electron-antineutrino disappearance at Daya bay," Physical Review Letters, vol. 108, no. 17, Article ID 171803, 2012.

[4] F. P. An, Q. An, J. Z. Bai et al., "Improved measurement of electron antineutrino disappearance at Daya Bay," Chinese Physics C, vol. 37, no. 1, Article ID 011001, 2013.

[5] J. K. Ahn, S. Chebotaryov, J. H. Choi et al., "Observation of reactor electron antineutrinos disappearance in the RENO experiment," Physical Review Letters, vol. 108, no. 19, Article ID 191802, 2012.

[6] K. Abe, N. Abgrall, H. Aihara et al., "Evidence of electron neutrino appearance in a muon neutrino beam," Physical Review D, vol. 88, no. 3, Article ID 032002, 2013.

[7] K. Abe, J. Adam, H. Aihara et al., "Observation of electron neutrino appearance in a muon neutrino beam," Physical Review Letters, vol. 112, no. 6, Article ID 061802, 2014.

[8] G. C. Branco, R. González Felipe, and F. R. Joaquim, "Leptonic CP violation," Reviews of Modern Physics, vol. 84, no. 2, pp. 515$565,2012$.

[9] H. Nunokawa, S. Parke, and J. W. F. Valle, "CP violation and neutrino oscillations," Progress in Particle and Nuclear Physics, vol. 60, no. 2, pp. 338-402, 2008.

[10] P. Adamson, D. J. Auty, D. S. Ayres et al., "Improved search for muon-neutrino to electron-neutrino oscillations in MINOS," Physical Review Letters, vol. 107, no. 18, Article ID 181802, 2011.

[11] P. Adamson, I. Anghel, C. Backhouse et al., "Measurement of neutrino and antineutrino oscillations using beam and atmospheric data in MINOS," Physical Review Letters, vol. 110, no. 25, Article ID 251801, 2013.

[12] Y. Abe, C. Aberle, T. Akiri et al., "Indication of reactor $\bar{\nu}_{e}$ disappearance in the double Chooz experiment," Physical Review Letters, vol. 108, no. 13, Article ID 131801, 2012.

[13] P. de Perio, K. Abe, C. Andreopoulos et al., "Oscillation results from T2K," http://arxiv.org/abs/1405.3871.

[14] D. Forero, M. Tortola, and J. Valle, "Global status of neutrino oscillation parameters after Neutrino-2012," Physical Review D, vol. 86, no. 7, Article ID 073012, 2012.

[15] G. L. Fogli, E. Lisi, A. Marrone, D. Montanino, A. Palazzo, and A. M. Rotunno, "Global analysis of neutrino masses, mixings, and phases: entering the era of leptonic $C P$ violation searches," Physical Review D, vol. 86, no. 1, Article ID 013012, 2012.

[16] M. C. Gonzalez-Garcia, M. Maltoni, J. Salvado, and T. Schwetz, "Global fit to three neutrino mixing: critical look at present 
precision," Journal of High Energy Physics, vol. 2012, no. 12, article 123, 2012.

[17] D. Forero, M. Tórtola, and J. W. F. Valle, "Neutrino oscillations refitted," Physical Review D, vol. 90, no. 9, Article ID 093006, 2014.

[18] P. Adamson, I. Anghel, A. Aurisano et al., "Combined analysis of $v_{\mu}$ disappearance and $v_{\mu} \rightarrow v_{e}$ appearance in MINOS using accelerator and atmospheric neutrinos," Physical Review Letters, vol. 112, no. 19, Article ID 191801, 2014.

[19] D. Ayres, J. W. Dawson, G. Drake et al., "NOvA proposal to build a 30 kilotonoff-axis detector to study neutrino oscillations in the fermilab NuMI beamline," http://arxiv.org/abs/hep-ex/ 0503053.

[20] R. Patterson, "Status of NOvA," in Proceedings of the Neutrino 2012 Conference, Kyoto, Japan, June 2012, http://neu2012.kek.jp/.

[21] S. K. Agarwalla, S. Prakash, S. K. Raut, and S. U. Sankar, "Potential of optimized $\mathrm{NO} \nu \mathrm{A}$ for large $\theta_{13} \&$ combined performance with a LArTPC \& T2K," Journal of High Energy Physics, vol. 2012, article 12, pp. 1-21, 2012.

[22] Y. Itow, T. Kajita, K. Kaneyuki et al., "The JHF-Kamiokaneutrino project," http://arxiv.org/abs/hep-ex/0106019.

[23] P. Huber, M. Lindner, and W. Winter, "Superbeams vs. neutrino factories," Nuclear Physics B, vol. 645, no. 1-2, pp. 3-48, 2002.

[24] M. Ishitsuka, T. Kajita, H. Minakata, and H. Nunokawa, "Resolving the neutrino mass hierarchy and $C P$ degeneracy by two identical detectors with different baselines," Physical Review D, vol. 72, no. 3, Article ID 033003, 2005.

[25] K. Abe, J. Adam, H. Aihara et al., "Neutrino oscillation physics potential of the T2K experiment," Progress of Theoretical and Experimental Physics, vol. 4, Article ID 043C01, 2015.

[26] K. Abe, H. Aihara, C. Andreopoulos et al., "A long baselineneutrino oscillation experiment using J-PARC neutrino beam and hyper-Kamiokande," http://arxiv.org/abs/1412.4673.

[27] E. K. Akhmedov, R. Johansson, M. Lindner, T. Ohlsson, and T. Schwetz, "Series expansions for three-flavor neutrino oscillation probabilities in matter," Journal of High Energy Physics, vol. 2004, no. 4, article 078, 2004.

[28] A. Cervera, A. Donini, M. Gavela et al., "Golden measurements at a neutrino factory," Nuclear Physics B, vol. 579, no. 1-2, pp. 17-55, 2000.

[29] M. Freund, "Analytic approximations for three neutrino oscillation parameters and probabilities in matter," Physical Review D, vol. 64, no. 5, Article ID 053003, 2001.

[30] P. Huber, M. Lindner, and W. Winter, "From parameter space constraints to the precision determination of the leptonic Dirac CP phase," Journal of High Energy Physics, vol. 2005, article 020, 2005.

[31] P. Huber, M. Lindner, T. Schwetz, and W. Winter, "First hint for $\mathrm{CP}$ violation in neutrino oscillations from upcoming superbeam and reactor experiments," Journal of High Energy Physics, vol. 2009, no. 11, article 044, 2009.

[32] A. Chatterjee, P. Ghoshal, S. Goswami, and S. K. Raut, "Octant sensitivity for large $\theta_{13}$ in atmospheric and long-baseline neutrino experiments," Journal of High Energy Physics, vol. 2013, no. 6, article 010, 2013.

[33] S. K. Agarwalla, S. Prakash, and S. Uma Sankar, "Resolving the octant of $\theta_{23}$ with T2K and NOvA," Journal of High Energy Physics, vol. 2013, no. 7, article 131, 2013.

[34] S. K. Agarwalla, "Physics potential of long-baseline experiments," Advances in High Energy Physics, vol. 2014, Article ID 457803, 29 pages, 2014.
[35] A. de Gouvêa, J. Jenkins, and B. Kayser, "Neutrino mass hierarchy, vacuum oscillations, and vanishing $\left|U_{e 3}\right|$," Physical Review D, vol. 71, Article ID 113009, 2005.

[36] S. K. Raut, "Effect of nonzero $\theta_{13}$ on the measurement of $\theta_{23}$ ", Modern Physics Letters A, vol. 28, no. 20, Article ID 1350093, 2013. 

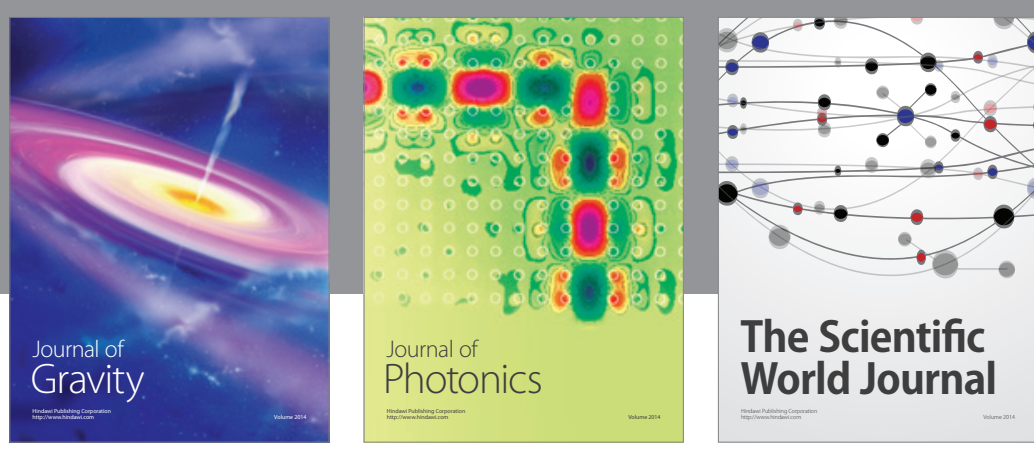

The Scientific World Journal
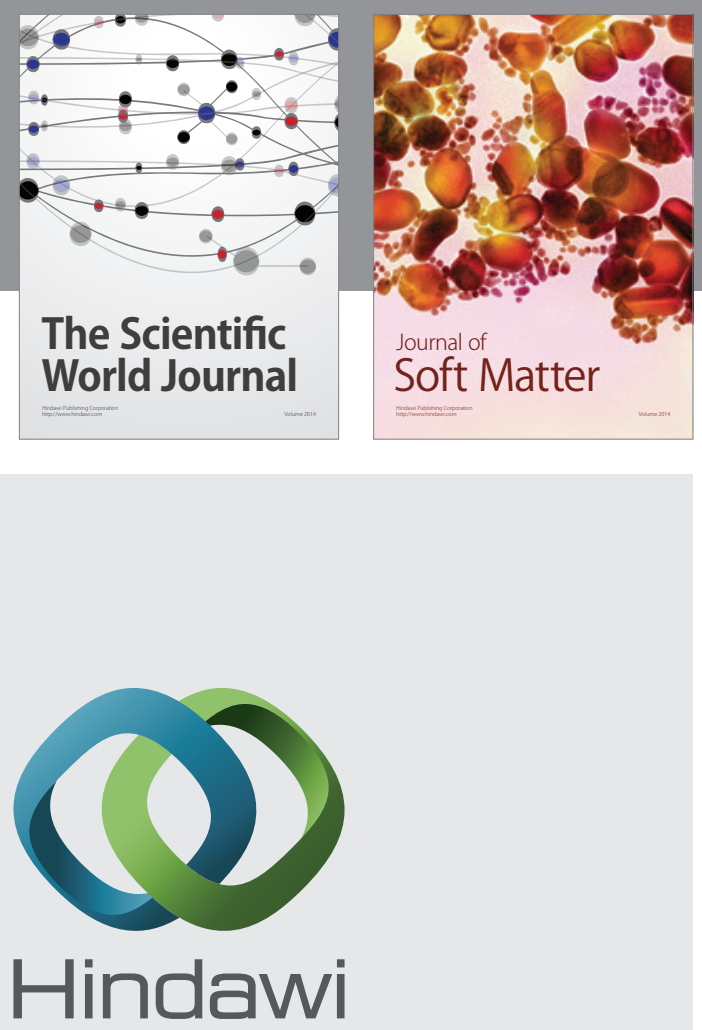

Submit your manuscripts at

http://www.hindawi.com

nternational Journal of

Statistical Mechanics
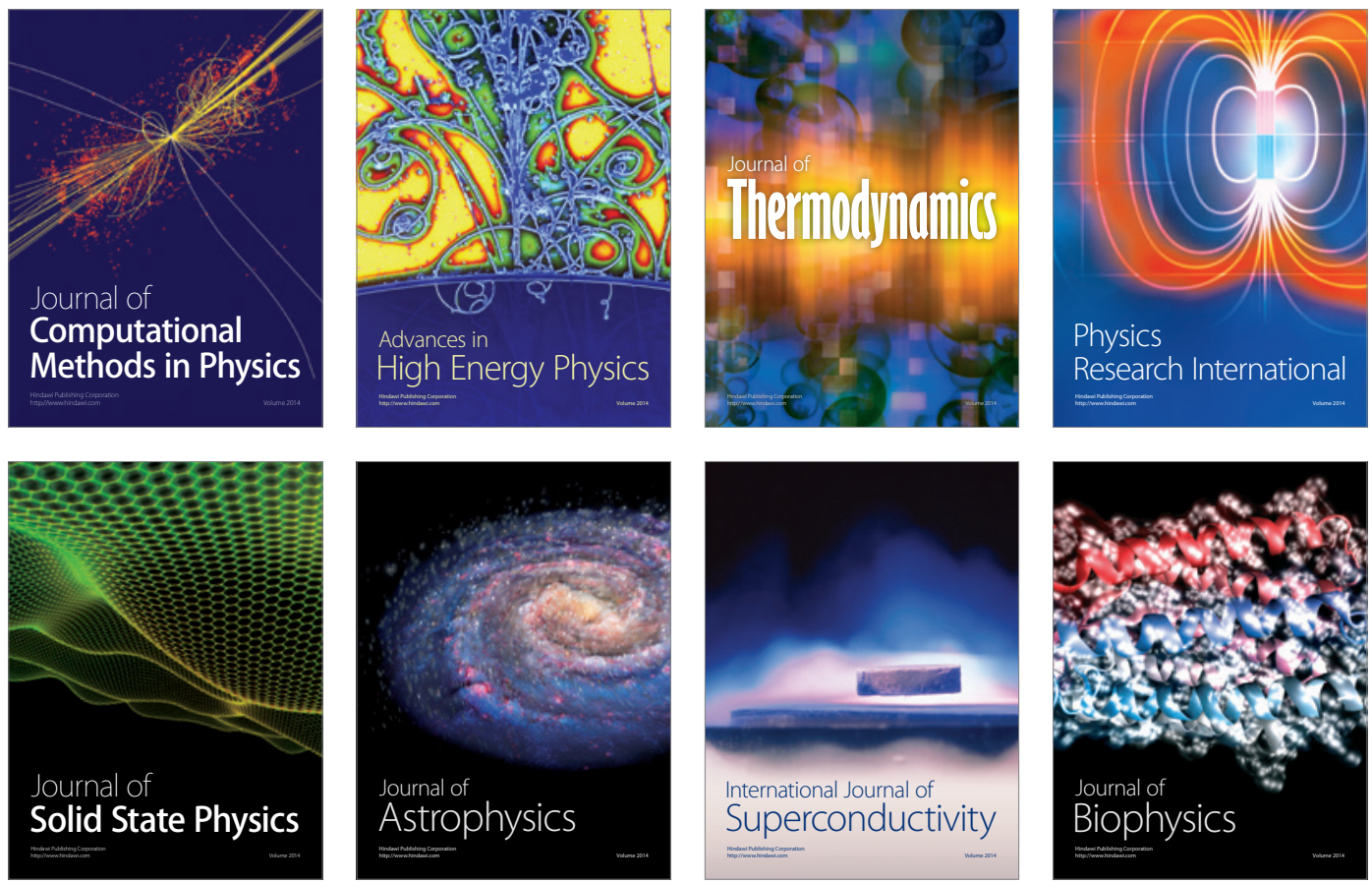
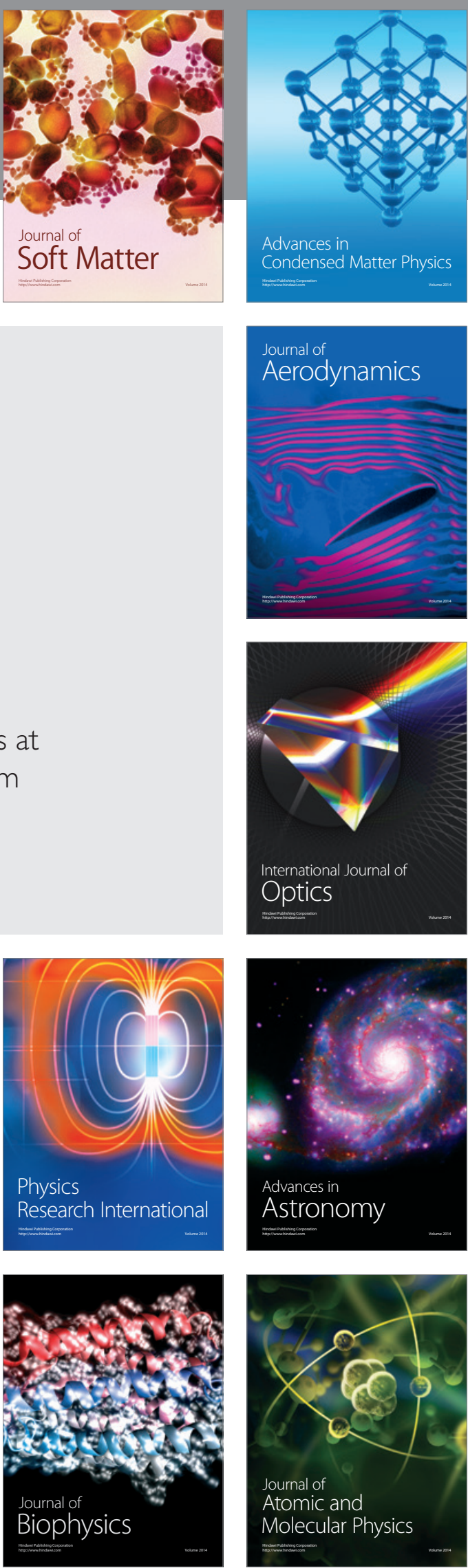